\title{
Non-autonomous stochastic Cauchy problems in Banach spaces
}

\author{
by \\ Mark Veraar (Delft) and Jan Zimmerschied (Karlsruhe)
}

\begin{abstract}
We study the non-autonomous stochastic Cauchy problem on a real Banach space $E$,

$$
\begin{aligned}
d U(t) & =A(t) U(t) d t+B(t) d W_{H}(t), \quad t \in[0, T], \\
U(0) & =u_{0} .
\end{aligned}
$$

Here, $W_{H}$ is a cylindrical Brownian motion on a real separable Hilbert space $H,(B(t))_{t \in[0, T]}$ are closed and densely defined operators from a constant domain $\mathcal{D}(B) \subset H$ into $E$, $(A(t))_{t \in[0, T]}$ denotes the generator of an evolution family on $E$, and $u_{0} \in E$. In the first part, we study existence of weak and mild solutions by methods of van Neerven and Weis. Then we use a well-known factorisation method in the setting of evolution families to obtain time regularity of the solution. In the second part, we consider the parabolic case in the setting of Acquistapace and Terreni. By means of a factorisation method in the spirit of Da Prato, Kwapień, and Zabczyk we obtain space-time regularity results for parabolic evolution families on Banach spaces. We apply this theory to several examples. In the last part, relying on recent results of Dettweiler, van Neerven, and Weis, we prove a maximal regularity result where the $A(t)$ are as in the setting of Kato and Tanabe.
\end{abstract}

1. Introduction. Let $E$ be a real Banach space, $H$ a separable real Hilbert space, and $T>0$ some finite time. Let $(\Omega, \mathcal{F}, P)$ be a complete probability space with a filtration $\left(\mathcal{F}_{t}\right)_{t \in[0, T]}$ which satisfies the usual conditions and on which we can define a cylindrical Brownian motion.

We study the non-autonomous stochastic Cauchy problem

$$
\begin{aligned}
d U(t) & =A(t) U(t) d t+B(t) d W_{H}(t), \quad t \in[0, T], \\
U(0) & =u_{0},
\end{aligned}
$$

where $(A(t), \mathcal{D}(A(t)))_{t \in[0, T]}$ is the generator of a strongly continuous evolution family on $E,(B(t))_{t \in[0, T]}$ are closed operators from a constant domain

2000 Mathematics Subject Classification: Primary 60H15; Secondary 35R60, 47D06.

Key words and phrases: stochastic Cauchy problem, non-autonomous equation, parabolic evolution equation, evolution family, mild solution, weak solution, cylindrical Brownian motion, factorisation method, space-time regularity, maximal regularity, $H^{\infty}$ calculus, $\gamma$-boundedness, type and cotype. 
$\mathcal{D}(B) \subset H$ into $E, W_{H}$ is a cylindrical Brownian motion on a real separable Hilbert space $H$, and $u_{0} \in E$. Note that $B$ may be unbounded, but $\mathcal{D}(B(t))=\mathcal{D}(B)$ is constant in time.

For the case of $(A(t))_{t \in[0, T]}$ independent of $t$ this equation has been studied in many papers (cf. $[8,9,13,14,19,33]$ and references therein). The case where $(A(t))_{t \in[0, T]}$ depends on time has been studied for instance in [12] by Da Prato, Iannelli and Tubaro, in [41] by Seidler, and in [24] by Krylov. Working in a Hilbert space setting and with constant domains $\mathcal{D}(A(t))$, in [12] time regularity of solutions is considered, whereas in [41] questions of space-time regularity are addressed in a special parabolic setting. In [24] the space regularity in $L^{p}$ for $p \in[2, \infty)$ of the solution has been investigated in the situation where each $A(t)$ is a second order elliptic operator on $\mathbb{R}^{n}$.

This paper is organised as follows. In Section 2 we recall some results on evolution families. In the parabolic case, some existence and regularity results in the setting of Acquistapace and Terreni [2] and the setting of Kato and Tanabe [42, Section 5.3] are presented. In the second part of Section 2, we briefly recall some results on $\gamma$-radonifying operators, $\gamma$-boundedness, and stochastic integration in Banach spaces.

In Section 3 the existence of mild and weak solutions of (1.1) is characterised and a factorisation method from Da Prato, Kwapień and Zabczyk [13], Millet and Smoleński [29], and [41] is extended to obtain sufficient conditions for path continuity of solutions.

In Section 4 we study the parabolic case of (1.1). There we mainly work under the assumptions of Acquistapace and Terreni [2]. The factorisation method for parabolic evolution families of [41] is extended to our setting. Under suitable conditions on $E, A(t)$, and $B(t)$ we show that the mild solution $U$ satisfies $t \mapsto(w-A(t))^{\delta} U(t) \in C^{\lambda}([0, T] ; E)$ for certain $\lambda>0$ and $\delta \geq 0$. The choices of $\lambda$ and $\delta$ are related to the constants in [2]. This extends results for autonomous equations in $[13,14,19]$. For completeness we note that under additional conditions on the Banach space $E$ (see [32]) one can extend the results of Sections 3 and 4 to the case where $B$ also depends on $\Omega$ in a suitable way.

In Section 5 the results are illustrated with two examples from $[1,40,47]$. At the end of Section 5 an example is given where $B$ is a fixed unbounded operator. This arises naturally in the context of spatio-temporal white noise problems. Consider the following stochastic partial differential equation:

$$
\begin{aligned}
\frac{\partial u}{\partial t}(t, x) & =L(t, x) u(t, x)+\frac{\partial w}{\partial t}(t, x), \quad x \in[0,1], t \in[0, T], \\
u(0, x) & =0, \quad x \in[0,1] \\
u(t, 0) & =u(t, 1)=0
\end{aligned}
$$


where

$$
L(t, x, D)=a_{2}(t, x) D^{2}+a_{1}(t, x) D+a_{0}(t, x)
$$

and the coefficients satisfy certain continuity properties. To model this equation one could take $H=L^{2}(0,1), E=L^{p}(0,1)$, and $B$ to be the identity mapping. Usually $p \in[1, \infty)$ is large and in that case $B$ is unbounded. We combine the results of Section 4 with ideas of Dettweiler, van Neerven, and Weis [19] to obtain a space-time regularity result for (1.2). In particular, we show that the solution $u$ is in the space $C^{\lambda}([0, T] \times[0,1])$ for all $\lambda \in(0,1 / 4)$.

In Section 6 we study maximal regularity for the solution of (1.1). This is done for the case where $B: H \rightarrow E$ is a fixed bounded operator which is $\gamma$-radonifying. In [19] it is shown that whenever $A$ admits a $\gamma$-bounded $H^{\infty}$-calculus, there exists a mild solution $U:[0, T] \times \Omega \rightarrow E$ such that $t \mapsto(-A)^{1 / 2} U(t) \in C\left([0, T] ; L^{p}(\Omega ; E)\right)$ for all $p \in[1, \infty)$. In the case where $E$ is a Hilbert space, a similar result was obtained in [14]. With regard to applications, maximal regularity can be used to study equations with additive noise and non-linear unbounded drift. Building on the results in [19], we will show that there exists a mild solution $U:[0, T] \times \Omega \rightarrow E$ such that $t \mapsto(-A(t))^{1 / 2} U(t) \in B\left([0, T] ; L^{p}(\Omega ; E)\right)$ for all $p \in[1, \infty)$, where $B([0, T] ; F)$ stands for the bounded and measurable functions from $[0, T]$ to $F$. This result is proved under the assumption that the $A(t)$ are as in $[42$, Section 5.3] and admit a $\gamma$-bounded $H^{\infty}$-calculus uniformly in $t \in[0, T]$.

\section{Preliminaries}

2.1. Evolution families. Let $(A(t), \mathcal{D}(A(t)))_{t \in[0, T]}$ be a family of closed and densely defined operators. Consider the non-autonomous Cauchy problem

$$
\begin{aligned}
\frac{d u}{d t}(t) & =A(t) u(t), \quad t \in[s, T], \\
u(s) & =x .
\end{aligned}
$$

We say that $u$ is a classical solution of (2.1) if $u \in C^{1}((s, T], E), u(t) \in$ $\mathcal{D}(A(t))$ for all $t \in(s, T], u(s)=x$, and $\frac{d u}{d t}(t)=A(t) u(t)$ for all $t \in(s, T]$. We call $u$ a strict solution of $(2.1)$ if $u \in C^{1}([s, T], E), u(t) \in \mathcal{D}(A(t))$ for all $t \in[s, T], u(s)=x$, and $\frac{d u}{d t}(t)=A(t) u(t)$ for all $t \in[s, T]$.

A family $(P(t, s))_{0 \leq s \leq t \leq T}$ of bounded operators on $E$ is referred to as a strongly continuous evolution family if

(1) $P(s, s)=I$ for all $s \in[0, T]$.

(2) $P(t, s)=P(t, r) P(r, s)$ for all $0 \leq s \leq r \leq t \leq T$.

(3) The mapping $\left\{(\tau, \sigma) \in[0, T]^{2}: \sigma \leq \tau\right\} \ni(t, s) \mapsto P(t, s)$ is strongly continuous. 
We say that such a family $(P(t, s))_{0 \leq s \leq t \leq T}$ solves $(2.1)\left(\right.$ on $\left.\left(Y_{s}\right)_{s \in[0, T]}\right)$ if $\left(Y_{s}\right)_{s \in[0, T]}$ are dense subspaces of $E$ and for all $0 \leq s \leq t \leq T$ we have $P(t, s) Y_{s} \subset Y_{t} \subset \mathcal{D}(A(t))$ and the function $t \mapsto P(t, s) x$ is a strict solution of (2.1) for every $x \in Y_{s}$.

In [36, 37], G. Nickel shows that well-posedness (i.e. existence, uniqueness, and continuous dependence on $\left.\left(Y_{s}\right)_{s \in[0, T]}\right)$ of $(2.1)$ is equivalent to the existence and uniqueness of a strongly continuous evolution semigroup that solves $(2.1)$ on $\left(Y_{s}\right)_{s \in[0, T]}$.

Throughout this paper $(A(t))_{t \in[0, T]}$ generates a unique evolution family $(P(t, s))_{0 \leq s \leq t \leq T}$ that solves $(2.1)$. In the literature many sufficient conditions for this can be found (cf. the monographs [2, 4, 27, 38, 42, 43]). In the next subsection we recall some results for the parabolic case of (2.1).

2.2. Parabolic evolution families. As before, let $(A(t), \mathcal{D}(A(t)))_{t \in[0, T]}$ be a family of closed and densely defined operators on a Banach space $E$. We will briefly discuss the setting of Acquistapace and Terreni (cf. [2]) and of Kato and Tanabe (cf. [42, Section 5.3]). Note that most of the results below have versions for non-densely defined $A(t)$ as well.

2.2.1. The (AT) hypotheses. We start with the theory of Acquistapace and Terreni. In fact they study a slightly more general setting. If $E$ is a real Banach space the assumptions below should be understood to concern the complexification of the objects under consideration. Condition (AT) consists of the following two conditions:

(AT1) $\quad A(t)$ are linear operators on a Banach space $E$ and there are constants $w \in \mathbb{R}, K \geq 0$, and $\phi \in(\pi / 2, \pi)$ such that $\Sigma(\phi, w) \subset \varrho(A(t))$ and for all $\lambda \in \Sigma(\phi, w)$ and $t \in[0, T]$

$$
\|R(\lambda, A(t))\| \leq \frac{K}{1+|\lambda-w|}
$$

(AT2) There are constants $L \geq 0$ and $\mu, \nu \in(0,1]$ with $\mu+\nu>1$ such that for all $\lambda \in \Sigma(\phi, w)$ and $s, t \in[0, T]$,

$$
\left\|A_{w}(t) R\left(\lambda, A_{w}(t)\right)\left(A_{w}(t)^{-1}-A_{w}(s)^{-1}\right)\right\| \leq L|t-s|^{\mu}(|\lambda|+1)^{-\nu} .
$$

Here $\Sigma(\phi, w)=\{w\} \cup\{\lambda \in \mathbb{C} \backslash\{w\}:|\arg (\lambda-w)| \leq \phi\}$ and $A_{w}(t)=A(t)-w$. Below it will be convenient to define $\kappa_{\mu, \nu}=\mu+\nu-1 \in(0,1]$.

These conditions have been extensively studied in the literature, where also many examples can be found. The first condition may be seen as analyticity uniformly in $t \in[0, T]$.

If (AT1) holds and the domains are constant: $\mathcal{D}(A(0))=\mathcal{D}(A(t)), t \in$ $[0, T]$, and $(A(t))_{t \in[0, T]}$ is Hölder continuous from $\mathcal{D}(A(0))$ to $E$ with exponent $\eta$, then (AT2) holds with $\mu=\eta$ and $\nu=1$ (see [2, Section 7]). The conditions in that case reduce to the conditions in the theory of Sobolevskiu and Tanabe for constant domains (cf. [27, 38, 42]). 
Under assumptions (AT1) and (AT2) the following result holds (see [2, Theorems 6.1-6.4] and [47, Theorem 2.1]).

THEOREM 2.1. If condition (AT) holds, then there exists a unique strongly continuous evolution family $(P(t, s))_{0 \leq s \leq t \leq T}$ that solves $(2.1)$ on $\mathcal{D}(A(s))$ and for all $x \in E, P(t, s) x$ is a classical solution of (2.1). Moreover, $(P(t, s))_{0 \leq s \leq t \leq T}$ is continuous on $0 \leq s<t \leq T$ and there exists a constant $C>0$ such that for every $0 \leq s<t \leq T$,

$$
\begin{aligned}
& \left\|(w-A(t))^{\theta} P(t, s)\right\| \leq C(t-s)^{-\theta} \quad \text { for } 0 \leq \theta \leq 1, \\
& \left\|P(t, s)-e^{(t-s) A(s)}\right\| \leq C(t-s)^{\kappa_{\mu, \nu} .}
\end{aligned}
$$

We also note that by [47, Theorem 2.3] there exists a constant $C>0$ such that for all $0 \leq s \leq t \leq T$,

$$
\left\|(w-A(t))^{\alpha} P(t, s)(w-A(s))^{-\alpha}-e^{(t-s) A(s)}\right\| \leq C(t-s)^{\kappa_{\mu, \nu}}
$$

for $\alpha \in(0,1]$. If $\alpha=0$, one recovers (2.3). Finally, we recall from [47, Theorem 2.1] that there is a constant $C>0$ such that for all $\theta \in(0, \mu)$ and all $x \in \mathcal{D}\left((w-A(t))^{\theta}\right)$,

$$
\left\|P(t, s)(w-A(s))^{\theta} x\right\| \leq C(\mu-\theta)^{-1}(t-s)^{-\theta}\|x\| .
$$

Below, we need estimates of the operator $(\partial P(t, s) / \partial s) A(s)$. Such estimates are studied in $[3$, Section 6$]$ by considering the adjoint operators $\left(A(t)^{*}\right)_{t \in[0, T]}$, whose existence is guaranteed by the density of each $\mathcal{D}(A(t))$. Note that $\mathcal{D}\left(A(t)^{*}\right)$ is not norm dense in general, but only $\sigma\left(E^{*}, E\right)$-dense. However, if $E$ is reflexive, Kato's result says that a sectorial operator always has norm dense domain (cf. [48, Section VIII.4]).

The assumptions in $[3$, Section 6$]$ are:

$(\mathrm{AT} 1)^{*} \quad A(t)$ are linear operators on a Banach space $E$ and there are constants $w \in \mathbb{R}, K \geq 0$ and $\phi \in(\pi / 2, \pi)$ such that $\Sigma(\phi, w) \subset$ $\varrho\left(A(t)^{*}\right)$ and for all $\lambda \in \Sigma(\phi, w)$ and $t \in[0, T]$,

$$
\left\|R\left(\lambda, A(t)^{*}\right)\right\| \leq \frac{K}{1+|\lambda-w|}
$$

(AT2) $^{*} \quad$ There are constants $L \geq 0$ and $\mu, \nu \in(0,1]$ with $\mu+\nu>1$ such that for all $\lambda \in \Sigma(\phi, w)$ and $s, t \in[0, T]$,

$$
\left\|A_{w}(t)^{*} R\left(\lambda, A_{w}(t)^{*}\right)\left(\left(A_{w}(t)^{*}\right)^{-1}-\left(A_{w}(s)^{*}\right)^{-1}\right)\right\| \leq L|t-s|^{\mu}(|\lambda|+1)^{-\nu} .
$$

Clearly, assumption (AT1) implies (AT1)*.

The following result is contained in [3, Theorem 6.4]. For two Banach spaces $E$ and $F$, we denote the space of bounded linear operators from $E$ to $F$ by $\mathcal{B}(E, F)$. 
THEOREM 2.2. Under assumptions (AT) and (AT2) ${ }^{*}$ there exists a family $(Q(t, s))_{0 \leq s \leq t \leq T}$ of operators in $\mathcal{B}(E)$ such that for all $0 \leq s<t \leq T$, $\frac{d P(t, s)}{d s}=Q(t, s) \quad$ and $\quad Q(t, s) x=-P(t, s) A(s) x \quad$ for all $x \in \mathcal{D}(A(s))$. Moreover, there is a constant $C>0$ such that for all $0 \leq s<t \leq T$,

$$
\|Q(t, s)\| \leq C(t-s)^{-1} .
$$

2.2.2. The (KT) hypotheses. Next we will briefly recall some results of Kato and Tanabe (cf. [42, Section 5.3]). We say that $(A(t))_{t \in[0, T]}$ satisfies (KT) if it satisfies (AT1) and the following conditions:

(KT1) The function $t \mapsto\left(-A_{w}(t)\right)^{-1}$ is continuously differentiable in $\mathcal{B}(E)$.

(KT2) There are constants $K>0$ and $\eta \in(0,1)$ such that for all $s, t \in$ $[0, T]$,

$$
\left\|\frac{d}{d t}\left(-A_{w}(t)\right)^{-1}-\frac{d}{d s}\left(-A_{w}(s)\right)^{-1}\right\| \leq K|t-s|^{\eta} .
$$

(KT3) There are constants $L>0$ and $\rho \in(0,1)$ such that for every $\lambda \in \Sigma(\phi, w)$ and $t \in[0, T]$,

$$
\left\|\frac{d}{d t} R(\lambda, A(t))\right\| \leq \frac{L}{1+|\lambda|^{\rho}} .
$$

The following theorem follows from [42, Theorem 5.3.3] and [43, Theorem $6.1]$.

TheOREM 2.3. Assume that (KT) holds. Then there exists a unique strongly continuous evolution family $(P(t, s))_{0 \leq s \leq t \leq T}$ that solves $(2.1)$ on $\mathcal{D}(A(s))$ and for all $x \in E, P(t, s) x$ is a classical solution of (2.1). Moreover, for all $0 \leq s<t \leq T$ the function $\partial P(t, s) / \partial s$ has a bounded extension $Q(t, s)$ and there is a constant $C$ such that for all $0 \leq s<t \leq T$,

$$
\|Q(t, s)\| \leq C(t-s)^{-1} .
$$

In [42] the following representation formula for $P$ is used:

$$
P(t, s)=e^{(t-s) A(t)}+\int_{s}^{t} e^{(t-\tau) A(t)} R(\tau, s) d \tau,
$$

where $(R(t, s))_{0 \leq s<t \leq T} \subset \mathcal{B}(E)$ can be estimated by

$$
\|R(t, s)\| \leq C(t-s)^{\rho-1}, \quad 0 \leq s<t \leq T .
$$

Remark 2.4. As can be seen from [2, Section 7], conditions (AT2) and (KT) without (AT1) are logically independent. 
2.3. $\gamma$-radonifying operators. Let $\left(g_{k}\right)_{k \geq 1}$ be an independent sequence of $\mathcal{N}(0,1)$-random variables and $\mathcal{H}$ a separable Hilbert space with orthonormal basis $\left(h_{k}\right)_{k \geq 1}$. An operator $R \in \mathcal{B}(\mathcal{H}, E)$ is called $\gamma$-radonifying if the series $\sum_{k} g_{k} R h_{k}$ converges in $L^{2}(\Omega ; E)$. The subspace of $\gamma$-radonifying operators will be denoted by $\gamma(\mathcal{H}, E)$. It becomes a Banach space by taking

$$
\|R\|_{\gamma(\mathcal{H}, E)}:=\left(\mathbb{E}\left\|\sum_{k} g_{k} R h_{k}\right\|^{2}\right)^{1 / 2}
$$

as norm. This defines an operator ideal in $\mathcal{L}(H, E)$. Moreover, an operator $R \in \mathcal{B}(\mathcal{H}, E)$ is $\gamma$-radonifying if and only if $R R^{*} \in \mathcal{B}\left(E^{*}, E\right)$ is the covariance operator of a centred Radon Gaussian measure $\mu$ on $(E, \mathfrak{B}(E))$ and in that case $\|R\|_{\gamma(\mathcal{H}, E)}^{2}=\int_{E}\|x\|^{2} d \mu(x)$.

In the case $\mathcal{H}=L^{2}(0, T)$ or $L^{2}(0, T ; H)$, the above operator-theoretic notion is applied via representability which we will explain now.

A function $\Phi:[0, T] \rightarrow \mathcal{B}(H, E)$ is said to belong scalarly to $L^{2}(0, T ; H)$ if for every $x^{*} \in E^{*}$ the function $t \mapsto \Phi(t)^{*} x^{*}$ belongs to $L^{2}(0, T ; H)$. We say that $\Phi$ is $H$-strongly measurable if the mapping $t \mapsto \Phi(t) h$ is strongly measurable for all $h \in H$. We define $\gamma(0, T ; H, E)$ to be the space of $H$ strongly measurable functions $\Phi:(0, T) \rightarrow \mathcal{B}(H, E)$ that are scalarly in $L^{2}(0, T ; H)$ and induce an operator $R_{\Phi} \in \gamma\left(L^{2}(0, T ; H), E\right)$ by

$$
R_{\Phi}(f):=\int_{0}^{T} \Phi(t) f(t) d t
$$

where the integral is defined as a Pettis integral (cf. [33]). In that case we let

$$
\|\Phi\|_{\gamma(0, T ; H, E)}:=\left\|R_{\Phi}\right\|_{\gamma\left(L^{2}(0, T ; H), E\right)} .
$$

If $E$ has type 2 then we know from [34, Lemma 6.1] that $L^{2}(0, T ; \gamma(H, E))$ injects canonically into $\gamma\left(L^{2}(0, T ; H), E\right)$ with norm estimate $\|\Phi\|_{\gamma(0, T ; H, E)}$ $\leq C_{2}\|\Phi\|_{L^{2}(0, T ; \gamma(H, E))}$, where $C_{2}$ is the type 2 constant of $E$ and the function $\Phi$ is in $L^{2}(0, T ; \gamma(H, E))$.

If $E$ is arbitrary but $\Phi$ has the special form $\Phi=f B$ for $f \in L^{2}(0, T)$ and $B \in \gamma(H, E)$ it is known that (cf. [19, Lemma 2.1])

$$
\|\Phi\|_{\gamma(0, T ; H, E)}=\|f\|_{L^{2}(0, T)}\|B\|_{\gamma(H, E)} .
$$

For more information on this topic we refer to $[6,22,44]$.

2.4. $\gamma$-boundedness. A set $\mathcal{T} \subset \mathcal{B}(E)$ is said to be $\gamma$-bounded if there exists a constant $M \geq 0$ such that

$$
\left(\mathbb{E}\left\|\sum_{n=1}^{N} \gamma_{n} T_{n} x_{n}\right\|^{2}\right)^{1 / 2} \leq M\left(\mathbb{E}\left\|\sum_{n=1}^{N} \gamma_{n} x_{n}\right\|^{2}\right)^{1 / 2}
$$


for all $N \geq 1$ and all sequences $\left(T_{n}\right)_{n=1}^{N} \subset \mathcal{T}$ and $\left(x_{n}\right)_{n=1}^{N} \subset E$. The least constant $M$ for which this estimate holds is called the $\gamma$-bound of $\mathcal{T}$, and denoted $\gamma(\mathcal{T})$. By the Kahane-Khinchin inequalities (see [26, Corollary 3.2]), the exponent 2 may be replaced by any exponent $p \in(0, \infty)$.

Replacing the Gaussian sequence by a Rademacher sequence we obtain the related notion of $R$-boundedness. In that case the $R$-bound will be denoted by $R(\mathcal{T})$. By a standard randomisation argument one can show that every $R$-bounded collection is $\gamma$-bounded. If $E$ has finite cotype, then both notions of boundedness coincide (see [20, Proposition 12.11 and Theorem 12.27]).

The concept of $R$-boundedness was introduced by Berkson and Gillespie in [5], but had already been used in the work of Bourgain [7]. $R$-boundedness was then thoroughly studied in [10] by Clément, de Pagter, Sukochev, and Witvliet. In [45] Weis proved a relation between $R$-boundedness and maximal $L^{p}$-regularity of Cauchy problems. For an extensive overview on the subject we refer to $[17,25]$.

The next important result is due to Kalton and Weis [22, Proposition 4.11] (also see [35]).

Proposition 2.5. Assume $\operatorname{dim}(H) \geq 1$. For a strongly continuous operator family $N:(0, T) \rightarrow \mathcal{B}(E)$ the following assertions are equivalent:

(1) The collection $\{N(t): t \in(0, T)\}$ is $\gamma$-bounded with constant $C$.

(2) For all $\Phi \in \gamma(0, T ; H, E)$ we have $N \Phi \in \gamma(0, T ; H, E)$ with

$$
\|N \Phi\|_{\gamma(0, T ; H, E)} \leq C\|\Phi\|_{\gamma(0, T ; H, E)} .
$$

2.5. Stochastic integration. We briefly recall some results from [33]. Let the family $\left(W_{H}(t)\right)_{t \geq 0}$ in $\mathcal{B}\left(H, L^{2}(\Omega, \mathcal{F}, P)\right)$ be a cylindrical Brownian motion, that is,

(1) for each $h \in H \backslash\{0\},\left(\|h\|^{-1} W_{H}(t) h\right)_{t \geq 0}$ is a standard real Brownian motion,

(2) for every $t_{1}, t_{2} \in[0, T]$ and $h_{1}, h_{2} \in H$ we have

$$
\mathbb{E}\left(W_{H}\left(t_{1}\right) h_{1} \cdot W_{H}\left(t_{2}\right) h_{2}\right)=\min \left(t_{1}, t_{2}\right)\left[h_{1}, h_{2}\right]_{H} .
$$

We will assume that all real Brownian motions $\left(W_{H}(t) h\right)_{t \geq 0}$ are $\left(\mathcal{F}_{t}\right)_{t \geq 0^{-}}$ adapted. Starting with step functions we can then define a stochastic integral with respect to $W_{H}$ for $H$-valued $L^{2}$-functions by standard methods. For a function $\Phi:[0, T] \rightarrow \mathcal{B}(H, E)$ that is scalarly in $L^{2}(0, T ; H)$ we say that $\Phi$ is stochastically integrable (on $[0, T]$ with respect to $W_{H}$ ) if there exists a $Y \in L^{2}(\Omega ; E)$ such that for each $x^{*} \in E^{*}$,

$$
\left\langle Y, x^{*}\right\rangle=\int_{0}^{T} \Phi(t)^{*} x^{*} d W_{H}(t) \quad P \text {-almost surely. }
$$


The random variable $Y$ is called the stochastic integral of $\Phi$, written

$$
Y=\int_{0}^{T} \Phi(t) d W_{H}(t) .
$$

This definition entails the following basic characterisation due to van Neerven and Weis [33, Theorem 4.2]. It extends the results in [9, 39].

Proposition 2.6. For an $H$-strongly measurable $\Phi:[0, T] \rightarrow \mathcal{B}(H, E)$ that is scalarly in $L^{2}(0, T ; H)$, the following assertions are equivalent:

(1) $\Phi \in \gamma(0, T ; H, E)$.

(2) $\Phi$ is stochastically integrable on $[0, T]$.

Moreover, the following isometry holds:

$$
\mathbb{E}\left\|\int_{0}^{T} \Phi(t) d W_{H}(t)\right\|^{2}=\|\Phi\|_{\gamma(0, T ; H, E)}^{2} .
$$

In Section 3 we want to study regularity properties of the process $(t, \omega) \mapsto$ $\left(\int_{0}^{t} P(t, s) B d W_{H}(s)\right)(\omega)$ and other processes of this form. We need such processes to be strongly measurable. The following lemma establishes their strong progressive measurability.

Lemma 2.7. Assume that $\Phi:[0, T]^{2} \rightarrow \mathcal{B}(H, E)$ is $H$-strongly measurable and for all $t \in[0, T], \Phi(t, \cdot)$ is scalarly in $L^{2}(0, T ; H)$. If for all $t \in[0, T]$, $s \mapsto \Phi(t, s)$ is stochastically integrable, then the process $\zeta:[0, T] \times \Omega \rightarrow E$ defined by

$$
\zeta(t)=\int_{0}^{t} \Phi(t, s) d W_{H}(s)
$$

has a strongly progressive modification.

The technical proof is omitted; it is based on techniques of $[15$, Section IV.30].

3. General existence and regularity results. Let $E, A(t), H, B(t)$, $W_{H}$, and $u_{0}$ be as in (1.1). The precise definition of the cylindrical Brownian motion $W_{H}$ can be found in Section 2.5. Recall that $B(t)$ is not necessarily bounded and $\mathcal{D}(B(t))=\mathcal{D}(B)$ is constant in time.

An $E$-valued process $\{U(t)\}_{t \in[0, T]}$ is called a mild solution of the problem (1.1) if for all $0 \leq s<t \leq T$ the operator $P(t, s) B(s): \mathcal{D}(B) \rightarrow E$ has a continuous extension to a bounded operator $P_{B}(t, s): H \rightarrow E$ and for all $t \in$ $[0, T]$ the $\mathcal{B}(H, E)$-valued process $s \mapsto P_{B}(t, s)$ is stochastically integrable on $(0, t)$ and

$$
U(t)=P(t, 0) u_{0}+\int_{0}^{t} P_{B}(t, s) d W_{H}(s)
$$


almost surely. When no confusion can arise we will write

$$
\int_{0}^{t} P(t, s) B(s) d W_{H}(s):=\int_{0}^{t} P_{B}(t, s) d W_{H}(s) .
$$

The uniqueness of a mild solution of (1.1) follows directly from the uniqueness of the evolution family. It is not true in general that (1.1) has a mild solution, even in the autonomous case and for one-dimensional timeindependent bounded operators $B$. In $[18,33]$ the authors constructed simple counterexamples on $C(K)$ and $L^{p}$ with $1 \leq p<2$ for which no mild solution exists.

The following obvious characterisation of the existence of mild solutions follows from Proposition 2.6.

Proposition 3.1. The following assertions are equivalent:

(1) The problem (1.1) has a mild solution.

(2) For all $t \in[0, T]$ the function $s \mapsto P(t, s) B(s)$ is in $\gamma(0, t ; H, E)$.

In $[14,33]$ the authors consider weak solutions of (1.1) for $A=A(t)$ and $B=B(t)$ independent of $t$ and $B \in \mathcal{B}(H, E)$.

An $E$-valued process $U$ is called a weak solution of (1.1) if the paths $t \mapsto U(t)$ are almost surely integrable and for all $x^{*} \in \mathcal{D}\left(A^{*}\right)$ and $t \in[0, T]$ we have almost surely

$$
\left\langle U(t), x^{*}\right\rangle-\left\langle u_{0}, x^{*}\right\rangle=\int_{0}^{t}\left\langle U(s), A^{*} x^{*}\right\rangle d s+W_{H}(t) B^{*} x^{*} .
$$

It is shown there that the concepts of weak solutions and mild solutions are equivalent. If $A(t)$ is time-dependent the definition of a weak solution does not make sense in general since $\bigcap_{t \in[0, T]} D\left(A(t)^{*}\right)$ may be empty. Below we give a definition of a weak solution where "the functionals depend on $t$ " as well. This is a well-known technique for equations like (2.1). It is easily seen that the definition coincides with the above definition in the case $A$ does not depend on time.

To motivate our definition of a weak solution we make a formal calculation. We rewrite (1.1) as

$$
\begin{aligned}
U^{\prime}(s) & =A(s) U(s)+\frac{B(s) d W_{H}(s)}{d s}, \quad s \in[0, T], \\
U(0) & =u_{0} .
\end{aligned}
$$

Let $t \in[0, T]$ be arbitrary and let $\varphi \in C^{1}\left([0, t] ; E^{*}\right)$ be such that for all $s \in[0, t]$ we have $\varphi(s) \in \mathcal{D}\left(A(s)^{*}\right)$, and $s \mapsto A(s)^{*} \varphi(s)$ is in $C\left([0, t] ; E^{*}\right)$. If we apply $\varphi(s)$ on both sides of $(3.1)$ and integrate over $[0, t]$ it follows that 
almost surely,

$$
\int_{0}^{t}\left\langle U^{\prime}(s), \varphi(s)\right\rangle d s=\int_{0}^{t}\left\langle U(s), A(s)^{*} \varphi(s)\right\rangle d s+\int_{0}^{t} B(s)^{*} \varphi(s) d W_{H}(s) .
$$

We may integrate by parts to conclude that almost surely,

$$
\begin{aligned}
\langle U(t), \varphi(t)\rangle-\left\langle u_{0}, \varphi(0)\right\rangle-\int_{0}^{t}\left\langle U(s), \varphi^{\prime}(s)\right\rangle d s & \\
& =\int_{0}^{t}\left\langle U(s), A(s)^{*} \varphi(s)\right\rangle d s+\int_{0}^{t} B(s)^{*} \varphi(s) d W_{H}(s) .
\end{aligned}
$$

Motivated by this, we introduce the following definition for $B:[0, T] \rightarrow$ $\mathcal{B}(H, E)$ such that for all $h \in H, s \mapsto B(s) h$ is strongly measurable and $\int_{0}^{T}\|B(s)\|^{2} d s<\infty$. Weak solutions for unbounded $B(t)$ are defined and characterised in Section 4. For $t \in[0, T]$, let

$$
\begin{aligned}
G_{t}:=\left\{\varphi \in C^{1}\left([0, t] ; E^{*}\right) \mid \forall s \in[0, t], \varphi(s)\right. & \in \mathcal{D}\left(A(s)^{*}\right) \\
& \text { and } \left.s \mapsto A(s)^{*} \varphi(s) \in C\left([0, t] ; E^{*}\right)\right\} .
\end{aligned}
$$

We call a process $(U(t))_{t \in[0, T]}$ a weak solution of (1.1) if almost surely $t \mapsto$ $U(t)$ is in $L^{1}(0, T ; E)$ and for all $t \in[0, T]$ and all $\varphi \in G_{t}$ the equation (3.2) holds almost surely.

To relate weak and mild solutions we need a large class of functions $\varphi$ as above. For this we introduce the following condition:

(C) For all $t \in[0, T]$, there is a $\sigma\left(E^{*}, E\right)$-sequentially dense subspace $F_{t}$ of $E^{*}$ such that for all $x^{*} \in F_{t}$ the function $\varphi(s):=P(t, s)^{*} x^{*}$ is in $C^{1}\left([0, t] ; E^{*}\right)$ and $\varphi(s) \in \mathcal{D}\left(A(s)^{*}\right)$ for all $s \in[0, t]$ and

$$
\frac{d}{d s} \varphi(s)=-A(s)^{*} \varphi(s) \text {. }
$$

If (AT) and (AT2)* hold, then (C) holds with $F_{t}=\mathcal{D}\left(\left(A(t)^{*}\right)^{2}\right)$. This follows from [2, Theorem 6.1]) and [3, p. 1176]. If $E$ is reflexive, by Kato's result, one may take $F_{t}=D\left(A(t)^{*}\right)$. If (KT) holds, then it holds as well for $\left(A(t)^{*}\right)_{t \in[0, T]}$, and as in the previous case, one can check that in the reflexive case (C) is satisfied with $F_{t}=D\left(A(t)^{*}\right)$ (cf. [43, Theorem 6.3]). For nonreflexive spaces we do not know if $(\mathrm{C})$ holds under condition $(\mathrm{KT})$. However, by [42, Theorem 5.3.2] a weak solution is always unique. If $A=A(s)$ is independent of $s$ and generates a strongly continuous semigroup, then (C) holds with $F=F_{t}=\mathcal{D}\left(A^{\odot}\right)$, where $A^{\odot}$ denotes the sun dual of $A$ (cf. [30]).

The following proposition gives the relation between a weak and a mild solution of (1.1). 
Proposition 3.2. Let $B:[0, T] \rightarrow \mathcal{B}(H, E)$ be such that for all $h \in H$, $t \mapsto B(t) h$ is strongly measurable and $\int_{0}^{T}\|B(t)\|^{2} d t<\infty$. Assume $(\mathrm{C})$ holds. For a process $U:[0, T] \times \Omega \rightarrow E$ the following assertions are equivalent:

(1) $U$ is a mild solution of (1.1) and almost surely $U \in L^{1}(0, T ; E)$.

(2) $U$ is a weak solution of (1.1).

In particular, a weak solution is unique.

Condition $(\mathrm{C})$ is only needed in $(2) \Rightarrow(1)$. The result may be proved similarly to [14, Theorem 5.4] and [33, Theorem 7.1].

The following result extends [13, Theorem 1], [29, Theorem 2.2], and [41, Theorem 1.2] in the special case of additive noise. The short proof given below is an obvious adaption of the arguments in [13].

THEOREM 3.3. If

$$
\sup _{t \in[0, T]}\left\|s \mapsto(t-s)^{-\alpha} P(t, s) B(s)\right\|_{\gamma(0, t ; H, E)}<\infty
$$

for some $\alpha \in(0,1 / 2)$, then for every $t \in[0, T]$, the mapping $[0, t] \ni s \mapsto$ $P(t, s) B(s)$ is stochastically integrable and

$$
[0, T] \ni t \mapsto \int_{0}^{t} P(t, s) B(s) d W_{H}(s)
$$

has a modification with continuous paths. In particular, there is a mild solution of (1.1) and it has a continuous version.

Proof. It follows from the assumption and Proposition 2.6 that $s \mapsto$ $(t-s)^{-\alpha} P(t, s) B(s)$ is stochastically integrable on $[0, t]$ for every $t \in[0, T]$. Hence, we may define $\zeta_{1}:[0, T] \times \Omega \rightarrow E$ as

$$
\zeta_{1}(t)=\int_{0}^{t}(t-s)^{-\alpha} P(t, s) B(s) d W_{H}(s) .
$$

By Proposition 2.6 and the Kahane-Khinchin inequalities we find that for every $1 \leq p<\infty$ and $t \in[0, T]$,

$$
\begin{aligned}
\mathbb{E}\left\|\zeta_{1}(t)\right\|^{p} & \simeq\left\|s \mapsto(t-s)^{-\alpha} P(t, s) B(s)\right\|_{\gamma(0, t ; H, E)}^{p} \\
& \leq \sup _{t \in[0, T]}\left\|s \mapsto(t-s)^{-\alpha} P(t, s) B(s)\right\|_{\gamma(0, t ; H, E)}^{p}<\infty .
\end{aligned}
$$

Since $\zeta_{1}$ is measurable by Lemma 2.7 , we may integrate over $[0, T]$ to obtain $\zeta_{1} \in L^{p}\left(0, T ; L^{p}(\Omega ; E)\right)$ for every $1 \leq p<\infty$. Now, Fubini's theorem implies that for all $p \in[1, \infty)$ and almost all $\omega \in \Omega, \zeta_{1}(\cdot, \omega) \in L^{p}(0, T ; E)$. Let us fix a version of $\zeta_{1}$, a number $p$ such that $\alpha p>1$, and a set $\Omega_{0}$ with $P\left(\Omega_{0}\right)=1$ such that $\zeta_{1}(\cdot, \omega) \in L^{p}(0, T ; E)$ for all $\omega \in \Omega_{0}$. 
The stochastic integrability assertion follows from [33, Corollary 4.4]. Hence, we may define $\zeta_{2}:[0, T] \times \Omega \rightarrow E$ as

$$
\zeta_{2}(t)=\int_{0}^{t} P(t, s) B(s) d W_{H}(s) .
$$

Lemma 2.7 shows that $\zeta_{2}$ is progressively measurable.

Next, we check that there exists a continuous version of $\zeta_{2}$. It is well known (cf. [13, Lemma 1] and [41, Lemma 2.1(i)]) that for $\alpha \in(0,1)$ and $p \in$ $(1, \infty)$ such that $p \alpha>1$ and $f \in L^{p}(0, T ; E)$, the function $R_{\alpha} f:[0, T] \rightarrow E$ defined by

$$
R_{\alpha} f(t)=\int_{0}^{t}(t-s)^{\alpha-1} P(t, s) f(s) d s
$$

is continuous and satisfies

$$
\left\|R_{\alpha} f\right\|_{C([0, T] ; E)} \leq C\|f\|_{L^{p}(0, T ; E)}
$$

for some constant $C>0$. Therefore, it is sufficient to show that for all $t \in[0, T]$ and almost all $\omega \in \Omega_{0}$ we have

$$
\zeta_{2}(t, \omega)=\frac{\sin (\pi \alpha)}{\pi}\left(R_{\alpha} \zeta_{1}(\cdot, \omega)\right)(t) .
$$

To check (3.4), fix $t \in[0, T]$. Strong measurability of both sides in (3.4) and the Hahn-Banach theorem show that it is sufficient to check that for all $x^{*} \in E^{*}$, almost surely we have

$$
\left\langle\zeta_{2}(t), x^{*}\right\rangle=\frac{\sin (\pi \alpha)}{\pi} \int_{0}^{t}\left\langle(t-s)^{\alpha-1} P(t, s) \zeta_{1}(s), x^{*}\right\rangle d s .
$$

This follows from a standard argument via the stochastic Fubini theorem (see [13]).

Next we will illustrate how Theorem 3.3 may be used if the noise is an $E$-valued Brownian motion. Consider

$$
\begin{aligned}
d U(t) & =A(t) U(t) d t+d W(t), \quad t \in[0, T], \\
U(0) & =u_{0},
\end{aligned}
$$

where $(A(t), \mathcal{D}(A(t)))_{t \in[0, T]}$ is as before, $W$ is an $E$-valued Brownian motion, and $u_{0} \in E$.

First we rewrite (3.5) in the form (1.1). Let $H$ be the reproducing kernel Hilbert space for the $E$-valued Gaussian random variable $W(1)$, and let $B: H \rightarrow E$ be the canonical inclusion operator. Then $B \in \gamma(H, E)$ and $W=B W_{H}($ see $[9,33])$.

We can extend [13, Theorem 2] to the non-autonomous setting. Without much effort the proof works for type 2 spaces as well, and we will present it in this setting. 
Corollary 3.4. Let $E$ be a type 2 Banach space and let $W=B W_{H}$ be a Brownian motion. Then there is a mild solution of problem (3.5) and it has a continuous modification.

Proof. The type 2 assumption and (2.8) imply that for all $\alpha \in(0,1 / 2)$ and $t \in[0, T]$,

$$
\begin{aligned}
\left\|s \mapsto(t-s)^{-\alpha} P(t, s) B\right\|_{\gamma(0, t ; H, E)}^{2} & \leq C_{2}^{2}\left\|s \mapsto(t-s)^{-\alpha} P(t, s) B\right\|_{L^{2}(0, t ; \gamma(H, E))}^{2} \\
& \leq C_{2}^{2} C^{2}\left\|s \mapsto(t-s)^{-\alpha} B\right\|_{L^{2}(0, t ; \gamma(H, E))}^{2} \\
& =C_{2}^{2} \widetilde{C}^{2} t^{-2 \alpha+1}\|B\|_{\gamma(H, E)}^{2} \\
& \leq C_{2}^{2} \widetilde{C}^{2} T^{-2 \alpha+1}\|B\|_{\gamma(H, E)}^{2} .
\end{aligned}
$$

The result now follows from Theorem 3.3.

Going beyond type 2 spaces requires some extra regularity of the evolution family.

Corollary 3.5. Let $W=B W_{H}$ be a Brownian motion. Assume that for all $0 \leq s<t \leq T, \partial P(t, s) / \partial s$ has a bounded extension $Q(t, s)$ and that there exists a constant $C>0$ such that for all $0 \leq s<t \leq T$,

$$
\|Q(t, s)\| \leq C(t-s)^{-1} .
$$

Then there is a mild solution of problem (3.5) and it has a continuous modification.

Note that condition (3.6) is satisfied in many situations under conditions (AT1), (AT2) and (AT2)*, and also under (KT) (cf. Theorems 2.2 and 2.3). Another condition for this may be found in [46, Theorem 1 and Remark].

Proof. Let $H$ and $B \in \gamma(H, E)$ be as in the section preceding Corollary 3.4. Let $\alpha \in(0,1 / 2)$ be arbitrary. Fix $\beta \in(\alpha, 1 / 2)$. Let $C$ be such that for all $s, t \in[0, T],\|P(t, s)\| \leq C$ and (3.6) holds. It follows from [25, Example $2.18]$ that for all $t \in[0, T]$ the set $\mathcal{P}(t):=\left\{(t-s)^{-\alpha+\beta} P(t, s): s \in[0, t]\right\}$ is $R$-bounded with

$$
\begin{aligned}
R(\mathcal{P}(t)) \leq & \left\|t^{-\alpha+\beta} P(t, 0)\right\| \\
& +\int_{0}^{t}\left[(\beta-\alpha)(t-s)^{-\alpha+\beta-1}\|P(t, s)\|+(t-s)^{-\alpha+\beta}\|Q(t, s)\|\right] d s \\
\leq & ((\beta-\alpha)+1) C \int_{0}^{t}(t-s)^{-\alpha+\beta-1} d s+C t^{-\alpha+\beta} \\
\leq & C\left(1+(\beta-\alpha)^{-1}\right) T^{-\alpha+\beta}+C T^{-\alpha+\beta}=: C_{1}<\infty .
\end{aligned}
$$


By Proposition 2.5 and (2.8) we have, for all $\alpha \in(0,1 / 2)$,

$$
\begin{aligned}
\left\|s \mapsto(t-s)^{-\alpha} P(t, s) B\right\|_{\gamma(0, t ; H, E)}^{2} & \leq C_{1}^{2}\left\|s \mapsto(t-s)^{-\beta} B\right\|_{\gamma(0, t ; H, E)}^{2} \\
& =C_{1}^{2}(-2 \beta+1)^{-1} t^{-2 \beta+1}\|B\|_{\gamma(H, E)}^{2} \\
& \leq C_{1}^{2}(-2 \beta+1)^{-1} T^{-2 \beta+1}\|B\|_{\gamma(H, E)}^{2}
\end{aligned}
$$

and the result follows from Theorem 3.3.

Finally, we discuss an example where the $A(t)$ are bounded.

ExAmple 3.6. Let $W=B W_{H}$ be a Brownian motion. Assume that $A(t) \in \mathcal{B}(E)$ for every $t \in[0, T]$ and $t \mapsto A(t)$ is continuous. Then there is a mild solution of problem (3.5) and it has a continuous modification.

Proof. By classical results (see [38, Section 5.1]), $(A(t))_{t \in[0, T]}$ generates a unique evolution family $(P(t, s))_{0 \leq s \leq t \leq T}$ that solves (2.1) on $E$. Moreover,

$$
Q(t, s):=\frac{\partial P(t, s)}{\partial s}=-P(t, s) A(s), \quad 0 \leq s \leq t \leq T,
$$

and hence $\partial P(t, s) / \partial s$ is uniformly continuous on $\{(t, s) \in[0, T]: s \leq t\}$. Now the result follows from Corollary 3.5.

Remark 3.7. In Corollaries 3.4 and 3.5 one could also consider timedependent $B:[0, T] \rightarrow \mathcal{L}(H, E)$. It is obvious from the proofs that it is sufficient to know that for some $\beta \in(0,1 / 2)$,

$$
\sup _{t \in[0, T]}\left\|s \mapsto(t-s)^{-\beta} B(s)\right\|_{\gamma(0, t ; H, E)}<\infty .
$$

Moreover, if $E$ has type 2, then by Hölder's inequality it is enough to assume that $B \in L^{p}(0, T ; \gamma(H, E))$ for some $p>2$.

4. Existence and regularity in the parabolic case. In this section we will study space and time regularity results in the parabolic case. Throughout this section we assume that (AT) from Section 2.2 holds for $(A(t))_{t \in[0, T]}$ with parameters $\mu$ and $\nu$. Recall that $\kappa_{\mu, \nu}=\mu+\nu-1 \in(0,1]$. It will be convenient to take the initial value to be 0 , so in this section we consider

$$
\begin{aligned}
d U(t) & =A(t) U(t) d t+B(t) d W_{H}(t), \quad t \in[0, T], \\
U(0) & =0,
\end{aligned}
$$

where $E, A(t), H, B(t)$, and $W_{H}$ are as in Section 3 .

We prove a version of [13, Lemma 2] and [41, Lemma 2.1(ii)] for arbitrary Banach spaces under the (AT) condition. A comparison with both results is made in Remark 4.2. Since the proof is different from that of [13, Lemma 2], and as it is the cornerstone of the results to follow, we include the full proof. 
Lemma 4.1. Let $\alpha \in(0,1), \delta \in[0,1]$, and $p \in[1, \infty)$ be such that $\alpha-1 / p-\delta>0$. For $f \in L^{p}(0, T ; E)$ define the function $R_{\alpha} f:[0, T] \rightarrow E$ as

$$
\left(R_{\alpha} f\right)(t)=\int_{0}^{t}(t-s)^{\alpha-1} P(t, s) f(s) d s .
$$

Then for every $f \in L^{p}(0, T ; E)$ we have $\left(R_{\alpha} f\right)(t) \in \mathcal{D}\left((w-A(t))^{\delta}\right)$ for all $t \in[0, T]$. Moreover, the mapping $t \mapsto(w-A(t))^{\delta}\left(R_{\alpha} f\right)(t)$ is $\lambda$-Hölder continuous and there is a constant $C>0$ such that for all $f \in L^{p}(0, T ; E)$,

$$
\left\|t \mapsto(w-A(t))^{\delta}\left(R_{\alpha} f\right)(t)\right\|_{C^{\lambda}([0, T] ; E)} \leq C\|f\|_{L^{p}(0, T ; E)}
$$

for any $\lambda>0$ satisfying

(1) $\lambda<\alpha-1 / p-\delta$ if $\alpha-1 / p-\delta \leq \kappa_{\mu, \nu}$

(2) $\lambda \leq \kappa_{\mu, \nu}$ if $\alpha-1 / p-\delta>\kappa_{\mu, \nu}$.

Proof. We already saw in the proof of Theorem 3.3 that the integral in (4.2) is well defined and that $R_{\alpha} f \in C([0, T] ; E)$. Moreover,

$$
(w-A(0))^{\delta}\left(R_{\alpha} f\right)(0)=0 .
$$

So to prove the lemma it is sufficient to show that there is a constant $C^{\prime}$ such that for all $f \in L^{p}(0, T ; E)$ and for all $0 \leq$ $s<t \leq T$,

$$
\begin{aligned}
\left\|(w-A(t))^{\delta}\left(R_{\alpha} f\right)(t)-(w-A(s))^{\delta}\left(R_{\alpha} f\right)(s)\right\|_{E} & \\
& \leq C^{\prime}|t-s|^{\lambda}\|f\|_{L^{p}(0, T ; E)} .
\end{aligned}
$$

We claim that for all $t \in[0, T]$ the mapping

$$
r \mapsto(t-r)^{\alpha-1}(w-A(t))^{\delta} P(t, r) f(r)
$$

is integrable on $[0, t]$ and

$$
(w-A(t))^{\delta}\left(R_{\alpha} f\right)(t)=\int_{0}^{t}(t-r)^{\alpha-1}(w-A(t))^{\delta} P(t, r) f(r) d r .
$$

To show this, note that for all $t \in(r, T]$ we have $P(t, r) f(r) \in \mathcal{D}(A(t))$ and $r \mapsto(w-A(t))^{\delta} P(t, r) f(r)$ is strongly measurable, so we only have to show that

$$
\int_{0}^{t}(t-r)^{\alpha-1}\left\|(w-A(t))^{\delta} P(t, r) f(r)\right\| d r<\infty .
$$

We will show something more general below. From (2.2) and Hölder's inequality we deduce that for every $s \in[0, t)$, 


$$
\begin{aligned}
& \int_{s}^{t}(t-r)^{\alpha-1}\left\|(w-A(t))^{\delta} P(t, r) f(r)\right\| d r \\
\leq & C \int_{s}^{t}(t-r)^{\alpha-1-\delta}\|f(r)\| d r \leq C\left(\int_{s}^{t}(t-r)^{(\alpha-1-\delta) p^{\prime}} d r\right)^{1 / p^{\prime}}\|f\|_{L^{p}(0, T ; E)} \\
= & C \frac{1}{\left((\alpha-1-\delta) p^{\prime}+1\right)^{1 / p^{\prime}}}(t-s)^{\alpha-1 / p-\delta}\|f\|_{L^{p}(0, T ; E)} .
\end{aligned}
$$

In particular, (4.5) and hence (4.4) hold.

For the proof of (4.3) we use (4.4) and split the integral into three parts,

$$
\begin{aligned}
\|(w- & A(t))^{\delta}\left(R_{\alpha} f\right)(t)-(w-A(s))^{\delta}\left(R_{\alpha} f\right)(s) \| \\
\leq & \left\|\int_{s}^{t}(t-r)^{\alpha-1}(w-A(t))^{\delta} P(t, r) f(r) d r\right\| \\
& +\left\|\int_{0}^{s}\left[(t-r)^{\alpha-1}-(s-r)^{\alpha-1}\right](w-A(t))^{\delta} P(t, r) f(r) d r\right\| \\
& +\left\|\int_{0}^{s}(s-r)^{\alpha-1}\left((w-A(t))^{\delta} P(t, r)-(w-A(s))^{\delta} P(s, r)\right) f(r) d r\right\| \\
= & : I_{1}+I_{2}+I_{3} .
\end{aligned}
$$

We estimate $I_{1}, I_{2}$, and $I_{3}$ separately. In particular, we show that each of the integrals exists absolutely. We already showed in (4.6) that

$$
I_{1} \leq C \frac{1}{\left((\alpha-1-\delta) p^{\prime}+1\right)^{1 / p^{\prime}}}(t-s)^{\alpha-1 / p-\delta}\|f\|_{L^{p}(0, T ; E)} .
$$

For $I_{2}$, note that by (2.2) for all $x \in E$ and $r \in[0, s)$ we have

$$
\begin{aligned}
\left((s-r)^{\alpha-1}-(t-r)^{\alpha-1}\right) \| & (w-A(t))^{\delta} P(t, r) x \| \\
\leq & C\left((s-r)^{\alpha-1}-(t-r)^{\alpha-1}\right)(t-r)^{-\delta}\|x\| \\
& \leq C\left((s-r)^{\alpha-1-\delta}-(t-r)^{\alpha-1-\delta}\right)\|x\| .
\end{aligned}
$$

It follows from this and Hölder's inequality that

$$
\begin{aligned}
I_{2} & \leq C \int_{0}^{s}\left((s-r)^{\alpha-1-\delta}-(t-r)^{\alpha-1-\delta}\right)\|f(r)\| d r \\
& \leq C\left(\int_{0}^{s}\left((s-r)^{\alpha-1-\delta}-(t-r)^{\alpha-1-\delta}\right)^{p^{\prime}} d r\right)^{1 / p^{\prime}}\|f\|_{L^{p}(0, T ; E)} .
\end{aligned}
$$


Using the estimate $(a-b)^{q} \leq a^{q}-b^{q}$ for $q \geq 1$ and $a \geq b \geq 0$ we obtain

$$
\begin{aligned}
I_{2} & \leq C\|f\|_{L^{p}(0, T ; E)}\left(\int_{0}^{s}(s-r)^{(\alpha-1-\delta) p^{\prime}}-(t-r)^{(\alpha-1-\delta) p^{\prime}} d r\right)^{1 / p^{\prime}} \\
& =\frac{C\|f\|_{L^{p}(0, T ; E)}}{\left((\alpha-1-\delta) p^{\prime}+1\right)^{1 / p^{\prime}}}\left(s^{\alpha-1-\delta+1 / p^{\prime}}+(t-s)^{\alpha-1-\delta+1 / p^{\prime}}-t^{\alpha-1-\delta+1 / p^{\prime}}\right) \\
& \leq \frac{C\|f\|_{L^{p}(0, T ; E)}}{\left((\alpha-1-\delta) p^{\prime}+1\right)^{1 / p^{\prime}}}(t-s)^{\alpha-1 / p-\delta}
\end{aligned}
$$

In order to estimate $I_{3}$ fix some $\eta \in(0, \alpha-1 / p-\delta)$. For $x \in E$ we have

$$
\begin{aligned}
&\left.\|(w-A(t))^{\delta} P(t, r)-(w-A(s))^{\delta} P(s, r)\right) x \| \\
&=\left\|\left[(w-A(t))^{\delta} P(t, s)-(w-A(s))^{\delta}\right] P(s, r) x\right\| \\
& \leq\left\|\left[(w-A(t))^{\delta} P(t, s)-(w-A(s))^{\delta} e^{(t-s) A(s)}\right] P(s, r) x\right\| \\
&+\left\|\left[(w-A(s))^{\delta} e^{(t-s) A(s)}-(w-A(s))^{\delta}\right] P(s, r) x\right\| \\
& \stackrel{\text { (i) }}{\leq} C(t-s)^{\kappa \mu, \nu}\left\|(w-A(s))^{\delta} P(s, r) x\right\| \\
&+C(t-s)^{\alpha-1 / p-\delta-\eta}\left\|(w-A(s))^{\alpha-1 / p-\eta} P(s, r) x\right\| \\
& \stackrel{(\text { ii) }}{\leq} C(t-s)^{\kappa_{\mu, \nu}}(s-r)^{-\delta}\|x\| \\
&+C(t-s)^{\alpha-1 / p-\delta-\eta}(s-r)^{-\alpha+1 / p+\eta}\|x\|,
\end{aligned}
$$

where for (i) we used (2.4) and the uniform analyticity (AT1), and for (ii) we used (2.2). It follows from Hölder's inequality that

$$
\begin{aligned}
I_{3} \leq & C(t-s)^{\kappa_{\mu, \nu}} \int_{0}^{s}(s-r)^{\alpha-1-\delta}\|f(r)\| d r \\
& +C(t-s)^{\alpha-1 / p-\delta-\eta} \int_{0}^{s}(s-r)^{-1 / p^{\prime}+\eta}\|f(r)\| d r \\
\leq & C(t-s)^{\kappa_{\mu, \nu}} \frac{1}{\left((\alpha-1-\delta) p^{\prime}+1\right)^{1 / p^{\prime}}} T^{(\alpha-1-\delta) p^{\prime}+1}\|f\|_{L^{p}(0, T ; E)} \\
& +C(t-s)^{\alpha-1 / p-\delta-\eta} \frac{1}{\left(p^{\prime} \eta\right)^{1 / p^{\prime}}} T^{\eta}\|f\|_{L^{p}(0, T ; E)} .
\end{aligned}
$$

Putting all the previous estimates together we get

$$
\begin{aligned}
\|(w- & A(t))^{\delta}\left(R_{\alpha} f\right)(t)-(w-A(s))^{\delta}\left(R_{\alpha} f\right)(s) \| \\
& \leq\left(C_{1}(t-s)^{\alpha-1 / p-\delta}+C_{2}\left[(t-s)^{\kappa \mu, \nu}+(t-s)^{\alpha-1 / p-\delta-\eta}\right]\right)\|f\|_{L^{p}(0, T ; E)},
\end{aligned}
$$

where the constants depend on $\eta, C, T, \alpha, p, \delta$ and on the constants in (AT1) and (AT2). Now the statement can easily be verified. 
REMARK 4.2. In [13, Lemma 2], for time-independent $A(t)$, also functions $f \in L^{p}(0, T, E)$ with $(w-A)^{\gamma} f \in L^{p}(0, T ; E)$ for some $\gamma \in[0,1]$ are considered. Using such functions, Da Prato, Kwapień and Zabczyk obtain a result with the extra parameter $\gamma$. The statement in Lemma 4.1 also has a version with such a $\gamma$. This may be proved using [40, Equation (2.13)].

In [41, Lemma 2.1(ii)] a similar result is proved for $D(A(t))$ constant in time and $A(t)$ satisfying the condition of Tanabe [42, Section 5.2]. In [41, Lemma 2.1(ii)] the choice of $\lambda$ is only restricted by $\alpha, p$, and $\delta$ and not by the parameter appearing in the assumption on $A(t)$. This is due to condition (P4) in [41], which states that for all $\tau \in(0,1)$ the fractional domain spaces coincide, i.e. $D\left((w-A(0))^{\tau}\right)=D\left((w-A(t))^{\tau}\right)$, with equivalent norm uniformly in $t \in[0, T]$. In Lemma 4.1 we do not need such an assumption. However, under the same set of assumptions as in [41, Lemma 2.1(ii)] with the same proofs the assertion of Lemma 2.1(ii) is true in arbitrary Banach spaces.

We can now prove the following space-time regularity result.

Theorem 4.3. Let $\alpha \in(0,1 / 2)$ and assume

$$
\sup _{t \in[0, T]}\left\|s \mapsto(t-s)^{-\alpha} P(t, s) B(s)\right\|_{\gamma(0, t ; H, E)}<\infty .
$$

Then there is a mild solution $U$ of (4.1) and it has a modification with paths that satisfy $t \mapsto(w-A(t))^{\delta} U(t) \in C^{\lambda}([0, T] ; E)$ for any $\lambda>0$ and $\delta \geq 0$ such that

$$
\alpha-\delta \leq \kappa_{\mu, \nu} \quad \text { and } \quad \lambda+\delta<\alpha,
$$

or

$$
\alpha-\delta>\kappa_{\mu, \nu} \quad \text { and } \quad \lambda \leq \kappa_{\mu, \nu}
$$

The condition (4.8) is more limiting than (4.7). If $\kappa_{\mu, \nu} \geq 1 / 2$ the condition $\alpha-\delta \leq \kappa_{\mu, \nu}$ in (4.7) is always satisfied. Recall that the operator $B(t)$ is not necessarily bounded.

Proof. We may define $\zeta_{1}:[0, T] \times \Omega \rightarrow E$ by

$$
\zeta_{1}(t)=\int_{0}^{t}(t-s)^{-\alpha} P(t, s) B(s) d W_{H}(s) .
$$

It follows from Proposition 2.6 and the Kahane-Khinchin inequalities that for all $1 \leq p<\infty$ and all $t \in[0, T]$,

$$
\begin{aligned}
\mathbb{E}\left\|\zeta_{1}(t)\right\|^{p} & \simeq\left\|s \mapsto(t-s)^{-\alpha} P(t, s) B(s)\right\|_{\gamma(0, t ; H, E)}^{p} \\
& \leq \sup _{r \in[0, T]}\left\|s \mapsto(r-s)^{-\alpha} P(r, s) B(s)\right\|_{\gamma(0, r ; H, E)}^{p}<\infty .
\end{aligned}
$$

From Lemma 2.7 we deduce that $\zeta_{1}$ is measurable, and we may integrate over $[0, T]$ to find that $\zeta_{1} \in L^{p}\left(0, T ; L^{p}(\Omega ; E)\right)$ for all $1 \leq p<\infty$. By the 
Fubini theorem it follows that $\zeta_{1}(\cdot, \omega) \in L^{p}(0, T ; E)$ for all $p \in[1, \infty)$ and almost all $\omega \in \Omega$.

Choose $\delta \geq 0$ and $\lambda>0$ according to (4.7). Let $p \in[1, \infty)$ be so large that $\lambda<\alpha-1 / p-\delta$. Let $\Omega_{0}$ with $P\left(\Omega_{0}\right)=1$ be such that $\zeta_{1}(\cdot, \omega) \in L^{p}(0, T ; E)$ for all $\omega \in \Omega_{0}$. We may apply the first part of Lemma 4.1 to deduce that $t \mapsto \frac{\pi}{\sin (\pi \alpha)}(w-A(t))^{\delta} R_{\alpha} \zeta_{1}(t, \omega)$ is $\lambda$-Hölder continuous for each $\omega \in \Omega_{0}$.

In the same way as in Theorem 3.3 one can see that $[0, t] \ni s \mapsto$ $P(t, s) B(s)$ is stochastically integrable. We define $\zeta_{2}:[0, T] \times \Omega \rightarrow E$ as

$$
\zeta_{2}(t)=\int_{0}^{t} P(t, s) B(s) d W_{H}(s) .
$$

As in the proof of Theorem 3.3 we may conclude that $\zeta_{2}$ has a modification such that $t \mapsto(w-A(t))^{\delta} \zeta_{2}(t)$ has paths in $C_{0}^{\lambda}([0, T] ; E)$.

Next, choose $\delta \geq 0$ and $\lambda>0$ according to (4.8). Let $p \in[1, \infty)$ be so large that $\alpha-\delta-1 / p>\kappa_{\mu, \nu}$. We can repeat the above arguments using the second part of Lemma 4.1.

This enables us to extend [13, Proposition 2] to the non-autonomous case in type 2 spaces.

Corollary 4.4. Let $E$ be a type 2 space and $B \in L^{p}(0, T ; \gamma(H, E))$ for some $p>2$. Then there exists a mild solution $U$ of (4.1) and it has a modification such that the paths satisfy $t \mapsto(w-A(t))^{\delta} U(t) \in C^{\lambda}([0, T] ; E)$ for every choice of $\lambda>0$ and $\delta \geq 0$ with

(1) $\lambda+\delta<1 / 2-1 / p$ if $\kappa_{\mu, \nu} \geq 1 / 2-1 / p$,

(2) $\delta<1 / 2-1 / p-\kappa_{\mu, \nu}$ and $\lambda \leq \kappa_{\mu, \nu}$ if $\kappa_{\mu, \nu}<1 / 2-1 / p$.

Notice that in $(2)$ the space regularity $0<\delta<1 / 2$ can be as close to $1 / 2-1 / p$ as we wish, since (AT1) and (AT2) hold for smaller $\mu$ and $\nu$ as well. The time regularity is however also limited by the values of $\mu$ and $\nu$.

Proof. As in Corollary 3.4 and Remark 3.7 one can show that

$$
\sup _{t \in[0, T]}\left\|s \mapsto(t-s)^{-\alpha} P(t, s) B(s)\right\|_{\gamma(0, t ; H, E)}<\infty
$$

for every $0<\alpha<1 / 2-1 / p$. Now (1) and (2) follow from Theorem 4.3.

For general Banach spaces we need again an additional assumption to obtain a space-time regularity result.

Corollary 4.5. Assume that (3.6) holds and let $B:[0, T] \rightarrow \mathcal{L}(H, E)$ be such that $\sup _{t \in[0, T]}\left\|(t-\cdot)^{-\alpha} B(\cdot)\right\|_{\gamma(0, t ; H, E)}<\infty$ for some $\alpha \in(0,1 / 2)$. Then there exists a mild solution $U$ of (4.1) and it has a modification such that the paths satisfy $t \mapsto(w-A(t))^{\delta} U(t) \in C^{\lambda}([0, T] ; E)$ for every choice of $\lambda>0$ and $\delta \geq 0$ with 
(1) $\lambda+\delta<\alpha$ if $\kappa_{\mu, \nu} \geq \alpha$,

(2) $\delta<\alpha-\kappa_{\mu, \nu}$ and $\lambda \leq \kappa_{\mu, \nu}$ if $\kappa_{\mu, \nu}<\alpha$.

By Proposition 3.2, the solution $U$ is the unique weak solution of (4.1) as well.

Proof. As in Corollary 3.5 and Remark 3.7 one can show that (4.9) holds for all $\alpha^{\prime}<\alpha$, so the result follows from Theorem 4.3.

REMARK 4.6. The results in Theorem 4.3 and Corollary 4.4 may be proved under conditions different than (AT) as well. Indeed, for each theory of evolution families for which the assertions of Theorem 2.1 and (2.4) hold for a certain $\kappa_{\mu, \nu}=\mu+\nu-1>0$, one can prove Lemma 4.1. This lemma is the main ingredient in the proofs.

In a theory of evolution families where some of the estimates (2.2), (2.3) or (2.4) are missing, it is still possible to prove space or time regularity via a version of Lemma 4.1 .

As explained in Remark 4.2, Theorem 4.3 and Corollaries 4.4 and 4.5 also have versions under condition $(\mathrm{P})$ from [41].

To end this section we extend the definition of a weak solution to the case where each $B(t): \mathcal{D}(B) \subset H \rightarrow E$ is a densely defined closed operator such that for all $h \in \mathcal{D}(B), t \mapsto B(t) h$ is strongly measurable. We note that although $\mathcal{D}(B(t))$ is assumed to be constant in time, $\mathcal{D}\left(B(t)^{*}\right)$ may vary. For $t \in[0, T]$ let

$$
\begin{aligned}
G_{t, B}:=\left\{\varphi \in G_{t} \mid \forall s \in[0, t), \varphi(s)\right. & \in \mathcal{D}\left(B(s)^{*}\right) \\
& \text { and } \left.s \mapsto B(s)^{*} \varphi(s) \in L^{2}(0, t ; H)\right\} .
\end{aligned}
$$

A process $(U(t))_{t \in[0, T]}$ is said to be a weak solution of (1.1) if almost surely $t \mapsto U(t)$ is in $L^{1}(0, T ; E)$ and, for all $t \in[0, T]$ and all $\varphi \in G_{t, B}$, the equality (3.2) holds almost surely. If each $B(s)$ is bounded and $\int_{0}^{T}\|B(s)\|^{2} d s<\infty$, then $G_{t, B}=G_{t}$ for all $t \in[0, T]$ and hence the definition coincides with that in Section 3.

Under condition (AT) and an extra assumption, again weak and mild solutions coincide.

Proposition 4.7. Assume (C) and (AT) hold, and there are constants $w \in \mathbb{R}, C>0$, and $\delta<1 / 2$ such that for all $t \in[0, T]$ and $h \in \mathcal{D}(B)$,

$$
\left\|(w-A(t))^{-\delta} B(t) h\right\| \leq C\|h\| .
$$

Assume that for all $h \in \mathcal{D}(B), t \mapsto B(t) h$ is strongly measurable. Then for a process $U:[0, T] \times \Omega \rightarrow E$ the following assertions are equivalent:

(1) $U$ is a mild solution of $(1.1)$ and $U \in L^{1}(0, T ; E)$ almost surely.

(2) $U$ is a weak solution of (1.1).

In particular, a weak solution is unique. 
Condition (C) is only needed in $(2) \Rightarrow(1)$. The proof is left to the reader. Notice that (4.10) ensures $\mathcal{D}\left(A(t)^{*}\right) \subset \mathcal{D}\left(\left((w-A(t))^{\delta}\right)^{*}\right) \subset \mathcal{D}\left(B(t)^{*}\right)$ for all $t \in[0, T]$. Indeed, for each $t \in[0, T]$ there is a constant $C(t)$ such that for all $x^{*} \in \mathcal{D}\left(\left((w-A(t))^{\delta}\right)^{*}\right)$ and $h \in \mathcal{D}(B)$,

$$
\left|\left\langle B(t) h, x^{*}\right\rangle\right|=\left|\left\langle(w-A(t))^{-\delta} B(t) h,\left((w-A(t))^{\delta}\right)^{*} x^{*}\right\rangle\right| \leq C(t)\|h\|\left\|x^{*}\right\| .
$$

Whenever $A(t)$ and $B(t)$ are independent of $t$, this shows that under condition (4.10) one can define a weak solution by (3.1) also for unbounded operators $B$ (cf. [19]).

5. Examples. In this section some applications of the results of Section 4 to stochastic partial differential equations are discussed.

As explained in Section 2.2, there are many examples of operator families $(A(t), \mathcal{D}(A(t)))_{t \in[0, T]}$ on Banach spaces that satisfy the conditions of Corollaries 4.4 and 4.5. The first two examples below are taken from $[1,40,47]$ and are applications of Corollaries 4.4 and 4.5, respectively. The last example is an application of Theorem 2.1 and illustrates how unbounded operators $B$ may be used in spatio-temporal white noise problems.

EXAmple 5.1. Consider

$$
\begin{aligned}
d u(t, x) & =A(t, x, D) u(t, x) d t+d w(x, t), \quad t \in(0, T], x \in S, \\
C(t, x, D) u(t, x) & =0, \quad t \in(0, T], x \in \partial S, \\
u(0, x) & =0, \quad x \in S .
\end{aligned}
$$

Here $S$ is a bounded domain in $\mathbb{R}^{n}$ with boundary of class $C^{2}$ and outer unit normal vector $n(x)$. Let $\partial S$ be the disjoint union of two closed (possibly empty) subsets $\Gamma_{0}, \Gamma_{1}$ and

$$
\begin{aligned}
& A(t, x, D)=\sum_{i, j=1}^{n} a_{i j}(t, x) D_{i} D_{j}+\sum_{i=1}^{n} a_{i}(t, x) D_{i}+a_{0}(t, x), \\
& C(t, x, D)=\sum_{i=1}^{n} c_{i}(t, x) D_{i}+c_{0}(t, x) .
\end{aligned}
$$

We assume that the coefficients are real and satisfy

$$
a_{i j}, a_{i}, a_{0} \in C^{\mu}([0, T] ; C(\bar{S})), \quad c_{i}, c_{0} \in C^{\mu}\left([0, T] ; C^{1}(\bar{S})\right)
$$

for $i, j=1, \ldots, n$ and a constant $1 / 2<\mu \leq 1$. Furthermore, let $\left(a_{i j}\right)$ be symmetric and uniformly elliptic, i.e. there is a constant $\kappa>0$ such that

$$
\sum_{i, j=1}^{n} a_{i j}(t, x) \xi_{i} \xi_{j} \geq \kappa|\xi|^{2}, \quad x \in \bar{S}, t \in[0, T], \xi \in \mathbb{R}^{n} .
$$

Finally, $c_{0}=1$ and $c_{i}=0$ on $\Gamma_{0}$ for all $i=1, \ldots, n$ and there is a $\beta>0$ such that for all $x \in \Gamma_{1}$ and $t \in[0, T], \sum_{i=1}^{n} c_{k}(t, x) n_{k}(x) \geq \beta$. 
The noise term is given by

$$
w(t, x)=\sum_{k \geq 1} b_{k}(t, x) w_{k}(t),
$$

where $b_{k}:[0, T] \times S \rightarrow \mathbb{R}$ for $k \geq 1$ are measurable functions and it is assumed that

$$
\int_{0}^{T}\left(\int_{S}\left(\sum_{k \geq 1} b_{k}^{2}(t, x)\right)^{q / 2} d x\right)^{r / q} d t<\infty,
$$

where $q \in[2, \infty)$ and $r \in(2, \infty)$ are fixed.

We model the problem (5.1) on $E_{p}=L^{p}(S)$ for $2 \leq p \leq q$ as a problem of the form (3.5). Here $A_{p}(t)$ is the realisation on $E_{p}$ of $A(t, x, D)$ with domain

$$
\mathcal{D}\left(A_{p}(t)\right)=\left\{f \in W^{2, p}(S) \mid C(t, \cdot, D) f=0 \text { on } \partial S\right\} .
$$

Then as explained in $[1,40,47],\left(A_{p}(t), \mathcal{D}\left(A_{p}(t)\right)\right)$ satisfies $(\mathrm{AT})$ with constants $\mu$ as above and $\nu=1 / 2$. Take $H=l^{2}$ with standard basis $\left(e_{k}\right)_{k \geq 1}$ and let $B:[0, T] \rightarrow \mathcal{B}\left(H, E_{p}\right)$ be defined as $B(t) h=\sum_{k>1}\left[h, e_{k}\right] b_{k}(t)$. This is well defined, and it follows from the Kahane-Khinchin inequalities and (5.4) that $B \in L^{r}\left(0, T ; \gamma\left(H, E_{p}\right)\right)$.

We are now in the situation of Corollary 4.4(2) and infer that (5.1) has a mild solution $U$ such that $t \mapsto(w-A(\cdot))^{\delta} U(t) \in C^{\lambda}\left([0, T] ; E_{p}\right)$ for every choice of $\lambda>0$ and $\delta \geq 0$ with

$$
\begin{aligned}
\lambda+\delta<1 / 2-1 / r & \text { if } \mu \geq 1-1 / r, \\
\delta<1-1 / r-\mu \text { and } \lambda \leq \mu-1 / 2 & \text { if } \mu<1-1 / r .
\end{aligned}
$$

By Proposition 3.2, $U$ is a weak solution as well.

Moreover, if each $b_{k}$ is constant in time, one may let $r$ tend to infinity to obtain regularity for all $\delta<1 / 2-\eta$ and $\lambda \leq \eta$, where $0<\eta<\mu-1 / 2$ is arbitrary.

In case $\Gamma_{1}=\emptyset$ one may take $\mu \in(0,1]$ and $\nu=1$. If $\mu \in(0,1 / 2)$, one may use Corollary 4.4(2) to obtain a solution

$$
U \in C^{\lambda}\left([0, T] ; \mathcal{D}\left((w-A(0))^{\delta}\right)\right)
$$

for any choice $\lambda>0$ and $\delta \geq 0$ with

$$
\begin{aligned}
\lambda+\delta<1 / 2-1 / r & \text { if } \mu \geq 1 / 2-1 / r, \\
\delta<1 / 2-1 / r-\mu \text { and } \lambda \leq \mu & \text { if } \mu<1 / 2-1 / r .
\end{aligned}
$$

Again if the $b_{k}$ are time-independent one may let $r \rightarrow \infty$.

If one chooses $p$ and $q$ large enough, then it follows via Sobolev imbedding that $U=0$ on $\partial S \times(0, T]$ and $U \in C^{\lambda}\left([0, T] ; C^{2 \delta}(\bar{S})\right)$ with $\lambda$ and $\delta$ as before.

Next we consider an application of Corollary 4.5. 
EXAmple 5.2. Consider

$$
\begin{aligned}
d u(t, x) & =A(t, x, D) u(t, x) d t+d w(t, x), \quad t \in(0, T], x \in S, \\
C(t, x, D) u(t, x) & =0, \quad t \in(0, T], x \in \partial S, \\
u(0, x) & =0, \quad x \in S .
\end{aligned}
$$

Here $S$ is a bounded domain in $\mathbb{R}^{n}$ with boundary of class $C^{2}$ and outer unit normal vector $n(x)$, and

$$
\begin{aligned}
& A(t, x, D)=\sum_{i, j=1}^{n} D_{i}\left(a_{i j}(t, x) D_{j}\right)+a_{0}(t, x), \\
& C(t, x, D)=\sum_{i, j=1}^{n} a_{i j}(t, x) n_{i}(x) D_{j} .
\end{aligned}
$$

We assume that the coefficients are real and satisfy

$$
\begin{aligned}
a_{i j} & \in C^{\mu}([0, T] ; C(\bar{S})), \quad a_{i j}(t, \cdot) \in C^{1}(\bar{S}), \quad D_{k} a_{i j} \in C([0, T] \times \bar{S}), \\
a_{0} & \in C^{\mu}\left([0, T], L^{n}(S)\right) \cap C([0, T] ; C(\bar{S}))
\end{aligned}
$$

for $i, j, k=1, \ldots, n, t \in[0, T]$, and a constant $\mu \in(1 / 2,1]$. Furthermore, let $\left(a_{i j}\right)$ be symmetric and let $(5.2)$ hold. The noise term is as in (5.3), where $b_{k}:[0, T] \times S \rightarrow \mathbb{R}$ for $k \geq 1$ are measurable and it is assumed that

$$
\sup _{t \in[0, T]} \int_{S}\left(\int_{0}^{T} \sum_{k \geq 1}(t-s)^{-2 \alpha} b_{k}^{2}(s, x) d s\right)^{q / 2} d x<\infty,
$$

where $q \in[2, \infty)$ and $\alpha \in(0,1 / 2)$ are fixed.

We model the problem (5.7) on $E=L^{p}(S)$ for $1<p \leq q$ as in Example 5.1 (see (5.5)). Then as explained in [1, 40,47], $\left(A_{p}(t), \mathcal{D}\left(A_{p}(t)\right)\right)$ satisfies (AT) and (AT2)* with constants $\mu$ as above and $\nu \in(1-\mu, 1 / 2)$ arbitrary. The function $B$ is defined as in Example 5.1. It follows from [31, Proposition 6.1] that $\sup _{t \in[0, T]}\left\|(t-\cdot)^{-\alpha} B(\cdot)\right\|_{\gamma(0, t ; H, E)}<\infty$.

Since (AT2)* holds, we may now apply Corollary $4.5(2)$ to conclude that (5.7) has a mild solution $U$ such that $t \mapsto(w-A(t))^{\delta} U(t) \in C^{\lambda}([0, T] ; E)$ for every choice of $\lambda>0$ and $\delta \geq 0$ with

$$
\begin{aligned}
\lambda+\delta<\alpha & \text { if } k_{\mu, \nu} \geq \alpha, \\
\delta<\alpha-k_{\mu, \nu} \text { and } \lambda \leq k_{\mu, \nu} & \text { if } k_{\mu, \nu}<\alpha .
\end{aligned}
$$

By Proposition 3.2, $U$ is the unique weak solution as well.

Moreover, if each $b_{k}$ is time-independent one may let $\alpha \uparrow 1 / 2$. This gives regularity for all $\delta<1 / 2-\eta$ and $\lambda \leq \eta$, where $0<\eta<\mu-1 / 2$ is arbitrary.

REMARK 5.3. An example similar to Example 5.2 with Dirichlet boundary condition works a well. In that case $\mu \in(0,1]$ and $\nu=1$ and via Sobolev imbedding one may obtain Hölder space-time regularity results. 
Next, we give an example with spatio-temporal white noise. This was our motivation to include results in Sections 3 and 4 for unbounded $B$ as well. We study a time-dependent version of examples in [14, Theorem 5.20] and [19, Section 5]. In [14, Theorem 5.20] the authors use eigenfunctions and eigenvalues in case $A$ is a self-adjoint operator. The method of $[19$, Section 5] works also for operators that are not necessarily self-adjoint. The main problem is to find, for given $B, H$, and $E$, a space $F$ such that the operator $B$ defined on $H$ takes its values in $F$ and is $\gamma$-radonifying. In [19, Section 5] this is solved by replacing $E$ by an extrapolation space $F$ of $E$ such that $B$ maps $H$ into $F$ and $B \in \gamma(H, F)$ as well.

We do not know if the above method can be extended to non-constant $A(t)$. However, it is possible to consider $B: \mathcal{D}(B) \subset H \rightarrow E$ as an unbounded operator. The following example is the time-dependent case of an example in [19].

EXAMPLE 5.4. Consider the following equation driven by spatio-temporal white noise:

$$
\begin{aligned}
\frac{\partial u}{\partial t}(t, x) & =L(t, x) u(t, x)+\frac{\partial w}{\partial t}(t, x), \quad x \in[0,1], t \in[0, T], \\
u(0, x) & =0, \quad x \in[0,1], \\
u(t, 0) & =u(t, 1)=0
\end{aligned}
$$

where

$$
L(t, x, D)=a_{2}(t, x) D^{2}+a_{1}(t, x) D+a_{0}(t, x) .
$$

Here the coefficients are real and satisfy $a_{2}, a_{1}, a_{0} \in C^{\mu}([0, T] ; C([0,1]))$ for some $\mu \in(1 / 4,1]$. Furthermore, there is a $\kappa>0$ such that $a_{2} \geq \kappa$ and we assume that $a_{2} \in C^{\varepsilon}([0,1] ; C([0, T]))$ for some $\varepsilon>0$.

This equation is modelled as (1.1) with unbounded $B$ on $E=L^{p}(0,1)$ with $p \in[2, \infty), A_{p}(t)=L(t, \cdot), \mathcal{D}(A(t))=W^{2, p}(0,1) \cap W_{0}^{1, p}(0,1), H=$ $L^{2}(0,1), \mathcal{D}(B)=L^{p}(0,1)$, and $B f=f$. As in Example 5.1 for $w$ sufficiently large $\left(A_{p}(t)-w\right)_{t \in[0, T]}$ satisfies (AT1) and the Tanabe conditions (see [42, Section 5.2]) with parameter $\mu$ and in particular (AT2) with $\mu$ and $\nu=1$ (see $[2$, Section 7]).

Next we check the conditions of Theorem 4.3. Let $0 \leq s<t \leq T$ and $\eta \in$ $(0, \mu)$ be fixed. It follows from $(2.5)$ that $P(t, s)(w-A(s))^{\eta}$ may be extended to a bounded operator $P_{\eta}(t, s)$ with $\left\|P_{\eta}(t, s)\right\| \leq C(\mu-\eta)^{-1}(t-s)^{-\eta}$.

For an invertible and sectorial operator $C$ and $\eta \in \mathbb{R}$ we denote by $E_{\eta}^{C}$ the fractional domain space. As in [19, Section 5] one can show that $B \in \gamma\left(H, E_{-\eta}^{\Delta}\right)$ for all $\eta>1 / 4$. Let $B_{1}: W^{2,2}(0,1) \rightarrow \mathcal{D}\left((-\Delta)^{1-\eta}\right)$ be the identity. It is shown in [19] that $(-\Delta)^{1-\eta} B_{1} \in \gamma\left(W^{2,2}(0,1), E\right)$.

Since $A(t)$ satisfies the assumptions of the Tanabe theory it follows from $\left[42\right.$, Section 5.2] that $\left\{(w-A(t))(w-A(s))^{-1}: s, t \in[0, T]\right\}$ is uniformly 
bounded in $\mathcal{B}(E)$ and $\mathcal{B}(H)$. In particular, this implies that $\mathcal{D}(A(t))=$ $\mathcal{D}(A(0))$ with equivalent norms uniformly in $t \in[0, T]$. Since $\mathcal{D}(A(0))=$ $\mathcal{D}(\Delta)$ with equivalent norms we conclude that $\mathcal{D}(A(t))=\mathcal{D}(\Delta)$ with equivalent norms uniformly in $t \in[0, T]$.

By the $\varepsilon$-Hölder continuity assumption it follows from [16] that each $w-A(t)$ has bounded imaginary powers and there exist constants $C, \gamma>0$ such that for all $t \in[0, T]$ we have $\left\|(w-A(t))^{\tau i}\right\| \leq C e^{\gamma|\tau|}$. Of course $-\Delta$ has bounded imaginary powers as well. Carefully inspecting the proof of $[28$, Theorem 4.2.6] we get

$$
\mathcal{D}\left((w-A(t))^{1-\eta}\right)=[E, \mathcal{D}(A(t))]_{1-\eta}=[E, \mathcal{D}(\Delta)]_{1-\eta}=\mathcal{D}\left((-\Delta)^{1-\eta}\right)
$$

with equivalent norms uniformly in $t \in[0, T]$. Therefore, we obtain

$$
\left\|(w-A(t))^{1-\eta} B_{1}\right\|_{\gamma\left(W^{2,2}(0,1), E\right)} \simeq\left\|(-\Delta)^{1-\eta} B_{1}\right\|_{\gamma\left(W^{2,2}(0,1), E\right)},
$$

with constants uniform in $t \in[0, T]$. By the right-ideal property for $\gamma$ radonifying operators we conclude

$$
\begin{aligned}
& \left\|(w-A(t))^{-\eta} B\right\|_{\gamma(H, E)}=\left\|(w-A(t))^{1-\eta} B_{1}(w-A(t))^{-1}\right\|_{\gamma(H, E)} \\
& \quad \leq\left\|(w-A(t))^{-1}\right\|_{\mathcal{B}\left(H, W^{2,2}(0,1)\right)}\left\|(w-A(t))^{1-\eta} B_{1}\right\|_{\gamma\left(W^{2,2}(0,1), E\right)} .
\end{aligned}
$$

Since $\left\|(w-A(t))^{-1}\right\|_{\mathcal{B}\left(H, W^{2,2}(0,1)\right)}$ is bounded uniformly in $t \in[0, T]$, we have proved that for all $t \in[0, T]$,

$$
B \in \gamma\left(H, E_{-\eta}^{A(t)-w}\right) \quad \text { with } \quad C_{B, \eta}:=\sup _{t \in[0, T]}\left\|(w-A(t))^{-\eta} B\right\|_{\gamma(H, E)}<\infty .
$$

It follows from the above results that for all $0 \leq s<t \leq T, P(t, s) B$ extends to a bounded operator from $H$ into $E$. Moreover, since $L^{p}$ has type 2, for $\eta, \alpha>0$ with $\eta \in(1 / 4, \mu)$ and $\eta+\alpha<1 / 2$, and all $t \in[0, T]$,

$$
\begin{aligned}
\| s \mapsto(t & -s)^{-\alpha} P(t, s) B\left\|_{\gamma(0, t ; H, E)} \leq C_{2}\right\| s \mapsto(t-s)^{-\alpha} P(t, s) B \|_{L^{2}(0, t ; \gamma(H, E))} \\
& \leq C_{2}\left\|s \mapsto(t-s)^{-\alpha} P(t, s)(w-A(s))^{\eta}(w-A(s))^{-\eta} B\right\|_{L^{2}(0, t ; \gamma(H, E))} \\
& \leq C_{2} C(\mu-\eta)^{-1} C_{B, \eta}\left\|s \mapsto(t-s)^{-\eta-\alpha}\right\|_{L^{2}(0, t)} \leq C_{\mu, \eta, \alpha, T}<\infty .
\end{aligned}
$$

We may apply Theorem 4.3(1) in the case of unbounded $B$ with arbitrary $\alpha \in(0,1 / 4)$ to get a mild solution

$$
U \in C^{\lambda}\left([0, T] ; \mathcal{D}\left((w-A(0))^{\delta}\right)\right)=C^{\lambda}\left([0, T] ; H_{0}^{2 \delta, p}(0,1)\right)
$$

for any choice $\lambda>0$ and $\delta \geq 0$ with $\lambda+\delta<1 / 4$ and $2 \delta>1 / p$. It follows from Proposition 4.7 that $U$ is also a weak solution. One may choose $p$ large enough and use a Sobolev imbedding theorem to find that $U \in$ $C^{\lambda}\left([0, T] ; C_{0}^{2 \delta^{\prime}}([0,1])\right)$, where $\lambda>0$ and $0 \leq \delta^{\prime}<\delta$ are as before. As in [19] one may conclude that (5.9) has a solution $U \in C^{\lambda}([0, T] \times[0,1])$ for all $\lambda \in(0,1 / 4)$ with $U(\cdot, 0) \equiv U(\cdot, 1) \equiv 0$. 
6. Maximal regularity. For $A(t)$ independent of time, several authors studied maximal regularity problems related to $(1.1)$. In $[13,14]$ in the case where $E$ is a Hilbert space the authors obtained sufficient conditions such that the solution $U$ of (4.1) has the property that for all $t \in[0, T], U(t)$ takes values in $\mathcal{D}\left((-A)^{1 / 2}\right)$ almost surely and $(-A)^{1 / 2} U$ is continuous in second moment. Such regularity results open the way to study certain non-linear stochastic partial differential equations driven by additive noise. In [19] these results have been extended to certain Banach spaces under the assumption that $-A$ admits a bounded $H^{\infty}$-calculus. The notion of a bounded $H^{\infty}$ calculus was introduced by Cowling, Doust, McIntosh, and Yagi in [11] and has been studied by many authors.

We will consider the maximal regularity question for $A(t)$ depending on $t$ and satisfying the assumptions of the Kato-Tanabe theory as explained in Section 2.2.

For precise information on the bounded $H^{\infty}$-calculus and the $\gamma$-bounded $H^{\infty}$-calculus we refer to $[17,21,22,23,25]$. We use the same notation as in [19]. Consider the following hypothesis:

$\left(H_{\gamma}^{\infty}\right) \quad$ There exist $C>0$ and $\phi \in[0, \pi / 2)$ such that for all $t \in[0, T]$, - $A_{w}(t)$ admits a $\gamma$-bounded $H^{\infty}$-calculus on $\Sigma_{\phi}$ whose $\gamma$-bound satisfies

$$
\gamma\left(\left\{\left\|f\left(-A_{w}(t)\right)\right\|:\|f\|_{H^{\infty}\left(\Sigma_{\phi}\right)} \leq 1\right\}\right) \leq C .
$$

The hypothesis $\left(H^{\infty}\right)$ is defined similarly, with the weaker notion of uniform boundedness. If $E$ has Pisier's property $(\alpha)$, then the hypotheses are equivalent (see [23, Theorem 5.3]).

We will now prove the following maximal regularity result. For a Banach space $F$, we write $B([0, T] ; F)$ for the Banach space of bounded measurable functions $f:[0, T] \rightarrow F$.

Theorem 6.1. Assume that $E$ has type 2 and that $(A(t))_{t \in[0, T]}$ satisfies (KT) and $\left(H_{\gamma}^{\infty}\right)$. If $B \in \gamma(H, E)$, then there exists a mild solution $U$ of (4.1) with continuous paths and for all $p \in[1, \infty)$ there exists a constant $C>0$ such that for all $t \in[0, T]$,

$$
\mathbb{E}\left\|(w-A(t))^{1 / 2} U(t)\right\|^{p} \leq C^{p}\|B\|_{\gamma(H, E)}^{p} .
$$

Moreover, for every $p \in[1, \infty)$ the function $(t, \omega) \mapsto(w-A(t))^{1 / 2} U(t, \omega)$ belongs to $B\left([0, T] ; L^{p}(\Omega ; E)\right)$ and it is strongly progressive.

It follows from Proposition 3.2 and the preceding text that $U$ is the unique weak solution of (4.1) as well. 
Proof. It follows from Theorem 2.3 and Corollary 3.5 that (4.1) has a mild solution

$$
U(t)=\int_{0}^{t} P(t, s) B d W_{H}(s) .
$$

To prove the first statement it suffices, by Proposition 2.6 and the KahaneKhinchin inequalities, to show that there is a constant $C>0$ independent of $t$ such that

$$
\left\|s \mapsto(w-A(t))^{1 / 2} P(t, s) B\right\|_{\gamma(0, t ; H, E)} \leq C\|B\|_{\gamma(H, E)} .
$$

To do so we may use (2.6) to write

$$
\begin{aligned}
\| s \mapsto(w-A(t))^{1 / 2} P(t, s) & B \|_{\gamma(0, t ; H, E)} \\
\leq & \left\|s \mapsto(w-A(t))^{1 / 2} e^{(t-s) A(t)} B\right\|_{\gamma(0, t ; H, E)} \\
& +\left\|s \mapsto(w-A(t))^{1 / 2} V(t, s) B\right\|_{\gamma(0, t ; H, E)},
\end{aligned}
$$

where $V(t, s)=\int_{s}^{t} e^{(t-\tau) A(t)} R(\tau, s) d \tau$ and $R$ is as in (2.7). Since $E$ has type 2 it has finite cotype and we infer from $\left(H_{\gamma}^{\infty}\right)$ and [19, Theorem 6.2 and Remark 6.3] that there is a constant $C>0$ such that for all $t \in[0, T]$,

$$
\left\|s \mapsto(w-A(t))^{1 / 2} e^{(t-s) A(t)} B\right\|_{\gamma(0, t ; H, E)} \leq C\|B\|_{\gamma(H, E)} .
$$

To estimate the other term we use the fact that $E$ has type 2 to obtain

$$
\begin{aligned}
& \left\|s \mapsto(w-A(t))^{1 / 2} V(t, s) B\right\|_{\gamma(0, t ; H, E)} \\
& \quad \leq C_{2}\left\|s \mapsto(w-A(t))^{1 / 2} V(t, s) B\right\|_{L^{2}(0, t ; \gamma(H, E))} .
\end{aligned}
$$

It follows from (2.7) that

$$
\left\|(w-A(t))^{1 / 2} e^{(t-\tau) A(t)} R(\tau, s)\right\| \leq C(t-\tau)^{-1 / 2}(\tau-s)^{\rho-1},
$$

where $C$ is a constant independent of $t, s, \tau$. By the definition of $V$ we obtain

$$
\begin{aligned}
\| s & \mapsto(w-A(t))^{1 / 2} V(t, s) B \|_{L^{2}(0, t ; \gamma(H, E))} \\
& =\left(\int_{0}^{t}\left\|\int_{s}^{t}(w-A(t))^{1 / 2} e^{(t-\tau) A(t)} R(\tau, s) B d \tau\right\|_{\gamma(H, E)}^{2} d s\right)^{1 / 2} \\
& \leq\left(\int_{0}^{t}\left(\int_{s}^{t}\left\|(w-A(t))^{1 / 2} e^{(t-\tau) A(t)} R(\tau, s) B\right\|_{\gamma(H, E)} d \tau\right)^{2} d s\right)^{1 / 2} \\
& \leq\left(\int_{0}^{t}\left(\int_{s}^{t} C(t-\tau)^{-1 / 2}(\tau-s)^{\rho-1}\|B\|_{\gamma(H, E)} d \tau\right)^{2} d s\right)^{1 / 2} \\
& =\left(\int_{0}^{t}\left(C(t-s)^{\rho-1 / 2} \frac{\Gamma(1 / 2) \Gamma(\rho)}{\Gamma(\rho+1 / 2)}\|B\|_{\gamma(H, E)}\right)^{2} d s\right)^{1 / 2} \leq \widetilde{C} T^{\rho}\|B\|_{\gamma(H, E)}
\end{aligned}
$$


for a certain constant $\widetilde{C}>0$. This proves the estimate. The final assertion follows from this and Lemma 2.7 .

For general Banach spaces a similar result can be proved under the restriction that the parameter $\rho$ of (KT2) satisfies $\rho \in(1 / 2,1]$. In [42, Section 5.3] a general example is discussed where $\rho=1$. There are however situations where $\rho=1 / 2$.

Theorem 6.2. Assume that $E$ has finite cotype and that $(A(t))_{t \in[0, T]}$ satisfies (KT) with $\rho \in(1 / 2,1)$ and $\left(H_{\gamma}^{\infty}\right)$. If $B \in \gamma(H, E)$, then there exists a mild solution $U$ of $(4.1)$ with continuous paths and for all $p \in[1, \infty)$ there exists a constant $C$ such that for all $t \in[0, T]$,

$$
\mathbb{E}\left\|(w-A(t))^{1 / 2} U(t)\right\|^{p} \leq C^{p}\|B\|_{\gamma(H, E)}^{p} .
$$

Moreover, for every $p \in[1, \infty)$ the function $(t, \omega) \mapsto(w-A(t))^{1 / 2} U(t, \omega)$ belongs to $B\left([0, T] ; L^{p}(\Omega ; E)\right)$ and is strongly progressive.

It follows from Proposition 3.2 and the text before it that $U$ is the unique weak solution of (4.1) as well.

Proof. The proof is the same as that of Theorem 6.1, except for the estimate of the $\gamma$-norm of $s \mapsto(w-A(t))^{1 / 2} V(t, s) B$. We first estimate $R$ from the definition of $V$.

As in [42, Section 5.3] we may write $R(t, s) B=\sum_{m \geq 1} R_{m}(t, s) B$, where we inductively define

$$
\begin{aligned}
R_{1}(t, s) & =\frac{1}{2 \pi i} \int_{\Gamma} e^{\lambda(t-s)} \frac{\partial}{\partial t} R(\lambda, A(t)-w) d \lambda, \\
R_{m}(t, s) & =\int_{s}^{t} R_{1}(t, \tau) R_{m-1}(\tau, s) d \tau .
\end{aligned}
$$

Here $\Gamma=\left\{r e^{k i \phi}: r \in[0, \infty), k= \pm 1\right\}$ for some $\phi \in(\pi / 2, \pi)$. It follows from (2.8) and (KT3) that

$$
\begin{aligned}
\| s & \mapsto R_{1}(t, s) B \|_{\gamma(0, t ; H, E)} \\
& \leq \sum_{k \in\{-1,1\}} \frac{1}{2 \pi} \int_{0}^{\infty}\left\|s \mapsto e^{r(t-s) \cos (k \phi)} \frac{\partial}{\partial t} R\left(r e^{k \phi i}, A(t)-w\right) B\right\|_{\gamma(0, t ; H, E)} d r \\
& \leq \sum_{k \in\{-1,1\}} \frac{\|B\|_{\gamma(H, E)}}{2 \pi} \int_{0}^{\infty}\left(\int_{0}^{t} e^{2 r(t-s) \cos (k \phi)} d s\right)^{1 / 2}\left\|\frac{\partial}{\partial t} R\left(r e^{k \phi i}, A(t)-w\right)\right\| d r \\
& \leq \frac{L\|B\|_{\gamma(H, E)}}{\pi} \int_{0}^{\infty}\left(\frac{1-e^{2 r t \cos (\phi)}}{-2 r \cos (\phi)}\right)^{1 / 2} \frac{1}{1+r^{\rho}} d r
\end{aligned}
$$




$$
\begin{aligned}
& =\frac{L\|B\|_{\gamma(H, E)}}{\pi} \int_{0}^{\infty}\left(\frac{1-e^{2 x \cos (\phi)}}{-2 x \cos (\phi)}\right)^{1 / 2} \frac{t^{\rho-1 / 2}}{t^{\rho}+x^{\rho}} d x \\
& \leq \frac{L\|B\|_{\gamma(H, E)}}{\pi} \int_{0}^{1} \frac{t^{\rho-1 / 2}}{t^{\rho}+x^{\rho}} d x+\frac{L\|B\|_{\gamma(H, E)}}{\pi(-2 \cos (\phi))^{1 / 2}} \int_{1}^{\infty} x^{-1 / 2} \frac{t^{\rho-1 / 2}}{t^{\rho}+x^{\rho}} d x .
\end{aligned}
$$

Since $\rho<1$ we have

$$
\int_{0}^{1} \frac{t^{\rho-1 / 2}}{t^{\rho}+x^{\rho}} d x \leq \int_{0}^{1} \frac{t^{\rho-1 / 2}}{x^{\rho}} d x=\frac{t^{\rho-1 / 2}}{1-\rho} .
$$

Since $\rho>1 / 2$, the other term may be estimated as

$$
\int_{1}^{\infty} x^{-1 / 2} \frac{t^{\rho-1 / 2}}{t^{\rho}+x^{\rho}} d x \leq \int_{1}^{\infty} x^{-1 / 2} \frac{t^{\rho-1 / 2}}{x^{\rho}} d x=\frac{t^{\rho-1 / 2}}{\rho-1 / 2} .
$$

We may conclude that

$$
\left\|s \mapsto R_{1}(t, s) B\right\|_{\gamma(0, t ; H, E)} \leq C\|B\|_{\gamma(H, E)} t^{\rho-1 / 2}
$$

where

$$
C=\frac{L}{\pi(1-\rho)}+\frac{L}{\pi(-2 \cos (\phi))^{1 / 2}(\rho-1 / 2)} .
$$

We claim that for $m \geq 1$,

$$
\left\|s \mapsto R_{m}(t, s) B\right\|_{\gamma(0, t ; H, E)} \leq C^{m} t^{m \rho-1 / 2} \frac{\Gamma^{m-1}(\rho) \Gamma(\rho+1 / 2)}{\Gamma(m \rho+1 / 2)} .
$$

We already saw that the result holds for $m=1$. For the other $m$, by induction $[42,5.54]$ and (2.7) we obtain

$$
\begin{aligned}
\| s \mapsto R_{m+1}( & t, s) B\left\|_{\gamma(0, t ; H, E)} \leq \int_{0}^{t}\right\| R_{1}(t, \tau)\|\| R_{m}(\tau, s) B \|_{\gamma(0, \tau ; H, E)} d \tau \\
& \leq \int_{0}^{t} C(t-\tau)^{\rho-1} C^{m} \tau^{m \rho-1 / 2} \frac{\Gamma^{m-1}(\rho) \Gamma(\rho+1 / 2)}{\Gamma(m \rho+1 / 2)} d \tau \\
& =C^{m+1} t^{(m+1) \rho-1 / 2} \frac{\Gamma(\rho) \Gamma(m \rho+1 / 2)}{\Gamma((m+1) \rho+1 / 2)} \frac{\Gamma^{m-1}(\rho) \Gamma(\rho+1 / 2)}{\Gamma(m \rho+1 / 2)} \\
& =C^{m+1} t^{(m+1) \rho-1 / 2} \frac{\Gamma^{m}(\rho) \Gamma(\rho+1 / 2)}{\Gamma((m+1) \rho+1 / 2)} .
\end{aligned}
$$

This proves the claim. 
From the claim we deduce

$$
\begin{aligned}
& \|s \mapsto R(t, s) B\|_{\gamma(0, t ; H, E)} \leq \sum_{m \geq 1}\left\|s \mapsto R_{m}(t, s) B\right\|_{\gamma(0, t ; H, E)} \\
& \quad \leq t^{\rho-1 / 2} \Gamma(\rho+1 / 2) \sum_{m \geq 1} C^{m} T^{(m-1) \rho} \frac{\Gamma^{m-1}(\rho)}{\Gamma(m \rho+1 / 2)}=: \widetilde{C} t^{\rho-1 / 2}
\end{aligned}
$$

for a certain constant $\widetilde{C}$. Hence there is a constant $\bar{C}$ such that for all $t \in[0, T]$,

$$
\begin{aligned}
\left\|s \mapsto(w-A(t))^{1 / 2} V(t, s) B\right\|_{\gamma(0, t ; H, E)} & \\
& =\int_{0}^{t}\left\|(w-A(t))^{1 / 2} e^{(t-\tau) A(t)}\right\|\|s \mapsto R(\tau, s) B\|_{\gamma(0, \tau ; H, E)} d \tau \\
& \leq \bar{C} \int_{0}^{t}(t-\tau)^{-1 / 2} \tau^{\rho-1 / 2} d \tau \leq \bar{C} t^{\rho} \frac{\Gamma(1 / 2) \Gamma(\rho+1 / 2)}{\Gamma(\rho+1)} .
\end{aligned}
$$

This is the required estimate.

Acknowledgements. The authors are grateful to Jan van Neerven, Roland Schnaubelt, and Peer Kunstmann for useful comments. Furthermore, they are indebted to Lutz Weis for encouraging their research on this subject. We thank the referee for his suggestion to consider time-dependent $B$. The first named author was supported by the Marie Curie Fellowship Program, the Netherlands Organization for Scientific Research (NWO) (639.032.201), and the Research Training Network "Evolution Equations for Deterministic and Stochastic Systems" (HPRN-CT-2002-00281). The second named author gratefully acknowledges the support by a grant from the Deutsche Forschungsgemeinschaft (We 2847/1-2).

\section{References}

[1] P. Acquistapace, Evolution operators and strong solutions of abstract linear parabolic equations, Differential Integral Equations 1 (1988), 433-457.

[2] P. Acquistapace and B. Terreni, A unified approach to abstract linear nonautonomous parabolic equations, Rend. Sem. Mat. Univ. Padova 78 (1987), 47-107.

[3] -, 一, Regularity properties of the evolution operator for abstract linear parabolic equations, Differential Integral Equations 5 (1992), 1151-1184.

[4] H. Amann, Linear and Quasilinear Parabolic Problems, Vol. I, Abstract Linear Theory, Monogr. Math. 89, Birkhäuser Boston, Boston, MA, 1995.

[5] E. Berkson and T. A. Gillespie, Spectral decompositions and harmonic analysis on UMD spaces, Studia Math. 112 (1994), 13-49. 
[6] V. I. Bogachev, Gaussian Measures, Math. Surveys Monogr. 62, Amer. Math. Soc., Providence, RI, 1998.

[7] J. Bourgain, Vector-valued singular integrals and the $H^{1}-B M O$ duality, in: Probability Theory and Harmonic Analysis (Cleveland, OH, 1983), Monogr. Textbooks Pure Appl. Math. 98, Dekker, New York, 1986, 1-19.

[8] Z. Brzeźniak, Stochastic partial differential equations in M-type 2 Banach spaces, Potential Anal. 4 (1995), 1-45.

[9] Z. Brzeźniak and J. M. A. M. van Neerven, Stochastic convolution in separable Banach spaces and the stochastic linear Cauchy problem, Studia Math. 143 (2000), 43-74.

[10] P. Clément, B. de Pagter, F. A. Sukochev, and H. Witvliet, Schauder decomposition and multiplier theorems, ibid. 138 (2000), 135-163.

[11] M. Cowling, I. Doust, A. McIntosh, and A. Yagi, Banach space operators with a bounded $H^{\infty}$ functional calculus, J. Austral. Math. Soc. Ser. A 60 (1996), 51-89.

[12] G. Da Prato, M. Iannelli, and L. Tubaro, On the path regularity of a stochastic process in a Hilbert space, defined by the Itô integral, Stochastics 6 (1981/82), 315322.

[13] G. Da Prato, S. Kwapień, and J. Zabczyk, Regularity of solutions of linear stochastic equations in Hilbert spaces, ibid. 23 (1987), 1-23.

[14] G. Da Prato and J. Zabczyk, Stochastic Equations in Infinite Dimensions, Encyclopedia Math. Appl. 44, Cambridge Univ. Press, Cambridge, 1992.

[15] C. Dellacherie and P.-A. Meyer, Probabilities and Potential, North-Holland Math. Stud. 29, North-Holland, Amsterdam, 1978.

[16] R. Denk, G. Dore, M. Hieber, J. Prüss, and A. Venni, New thoughts on old results of R. T. Seeley, Math. Ann. 328 (2004), 545-583.

[17] R. Denk, M. Hieber, and J. Prüss, R-boundedness, Fourier multipliers and problems of elliptic and parabolic type, Mem. Amer. Math. Soc. 166 (2003), no. 788.

[18] J. Dettweiler and J. M. A. M. van Neerven, Continuity versus nonexistence for a class of linear stochastic Cauchy problems driven by a Brownian motion, Czechoslovak Math. J. 56 (131) (2006), 579-586.

[19] J. Dettweiler, J. M. A. M. van Neerven, and L. Weis, Space-time regularity of solutions of the parabolic stochastic Cauchy problem, Stoch. Anal. Appl. 24 (2006), 843-869.

[20] J. Diestel, H. Jarchow, and A. Tonge, Absolutely Summing Operators, Cambridge Stud. Adv. Math. 43, Cambridge Univ. Press, Cambridge, 1995.

[21] M. Haase, The Functional Calculus for Sectorial Operators, Oper. Theory Adv. Appl. 169, Birkhäuser, Basel, 2006.

[22] N. J. Kalton and L. Weis, The $H^{\infty}$-calculus and square function estimates, in preparation.

[23] - - - The $H^{\infty}$-calculus and sums of closed operators, Math. Ann. 321 (2001), 319-345.

[24] N. V. Krylov, An analytic approach to SPDEs, in: Stochastic Partial Differential Equations: Six Perspectives, Math. Surveys Monogr. 64, Amer. Math. Soc., Providence, RI, 1999, 185-242.

[25] P. C. Kunstmann and L. Weis, Maximal $L_{p}$-regularity for parabolic equations, Fourier multiplier theorems and $H^{\infty}$-functional calculus, in: Functional Analytic Methods for Evolution Equations, Lecture Notes in Math. 1855, Springer, Berlin, 2004, 65-311. 
[26] M. Ledoux and M. Talagrand, Probability in Banach Spaces, Ergeb. Math. Grenzgeb. 23, Springer, Berlin, 1991.

[27] A. Lunardi, Analytic Semigroups and Optimal Regularity in Parabolic Problems, Progr. Nonlinear Differential Equations Appl. 16, Birkhäuser, Basel, 1995.

[28] —, Interpolation Theory, Appunti, Scuola Norm. Sup. Pisa, 1999.

[29] A. Millet and W. Smoleński, On the continuity of Ornstein-Uhlenbeck processes in infinite dimensions, Probab. Theory Related Fields 92 (1992), 529-547.

[30] J. M. A. M. van Neerven, The Adjoint of a Semigroup of Linear Operators, Lecture Notes in Math. 1529, Springer, Berlin, 1992.

[31] J. M. A. M. van Neerven, M. C. Veraar, and L. Weis, Conditions for stochastic integrability in UMD Banach spaces, in: Banach Spaces and their Applications in Analysis (in Honor of Nigel Kalton's 60th Birthday), de Gruyter Proc. Math., de Gruyter, 2007, 127-146.

[32] - , - - Stochastic integration in UMD Banach spaces, Ann. Probab. 35 (2007), $1438-1478$.

[33] J. M. A. M. van Neerven and L. Weis, Stochastic integration of functions with values in a Banach space, Studia Math. 166 (2005), 131-170.

[34] - - - Weak limits and integrals of Gaussian covariances in Banach spaces, Probab. Math. Statist. 25 (2005), 55-74.

[35] - - - Invariant measures for the linear stochastic Cauchy problem and R-boundedness of the resolvent, J. Evol. Equ. 6 (2006), 205-228.

[36] G. Nickel, On evolution semigroups and wellposedness of nonautonomous Cauchy problems, Ph.D. thesis, Univ. Tübingen, 1996.

[37] - Evolution semigroups for nonautonomous Cauchy problems, Abstr. Appl. Anal. 2 (1997), 73-95.

[38] A. Pazy, Semigroups of Linear Operators and Applications to Partial Differential Equations, Appl. Math. Sci. 44, Springer, New York, 1983.

[39] J. Rosiński and Z. Suchanecki, On the space of vector-valued functions integrable with respect to the white noise, Colloq. Math. 43 (1980), 183-201 (1981).

[40] R. Schnaubelt, Asymptotic behaviour of parabolic nonautonomous evolution equations, in: Functional Analytic Methods for Evolution Equations, Lecture Notes in Math. 1855, Springer, Berlin, 2004, 401-472.

[41] J. Seidler, Da Prato-Zabczyk's maximal inequality revisited. I, Math. Bohem. 118 (1993), 67-106.

[42] H. Tanabe, Equations of Evolution, Monogr. Stud. Math. 6, Pitman (Advanced Publishing Program), Boston, MA, 1979.

[43] —, Functional Analytic Methods for Partial Differential Equations, Monogr. Textbooks Pure Appl. Math. 204, Dekker, New York, 1997.

[44] N. N. Vakhania, V. I. Tarieladze, and S. A. Chobanyan, Probability Distributions on Banach Spaces, Math. Appl. (Soviet Ser.) 14, Reidel, Dordrecht, 1987.

[45] L. Weis, Operator-valued Fourier multiplier theorems and maximal $L_{p}$-regularity, Math. Ann. 319 (2001), 735-758.

[46] A. Yagi, On the abstract evolution equation of parabolic type, Osaka J. Math. 14 (1977), 557-568.

[47] -, Abstract quasilinear evolution equations of parabolic type in Banach spaces, Boll. Un. Mat. Ital. B (7) 5 (1991), 341-368. 
[48] K. Yosida, Functional Analysis, 6th ed., Grundlehren Math. Wiss. 123, Springer, Berlin, 1980.

Delft Institute of Applied Mathematics Technical University of Delft

P.O. Box 5031

2600 GA Delft, The Netherlands

E-mail: M.C.Veraar@math.tudelft.nl mark@profsonline.nl
Institut für Analysis Universität Karlsruhe (TH)

Englerstraße 2

76128 Karlsruhe, Germany

E-mail: Jan.Zimmerschied@HVB.de

Received September 27, 2005

Revised version December 31, 2007 